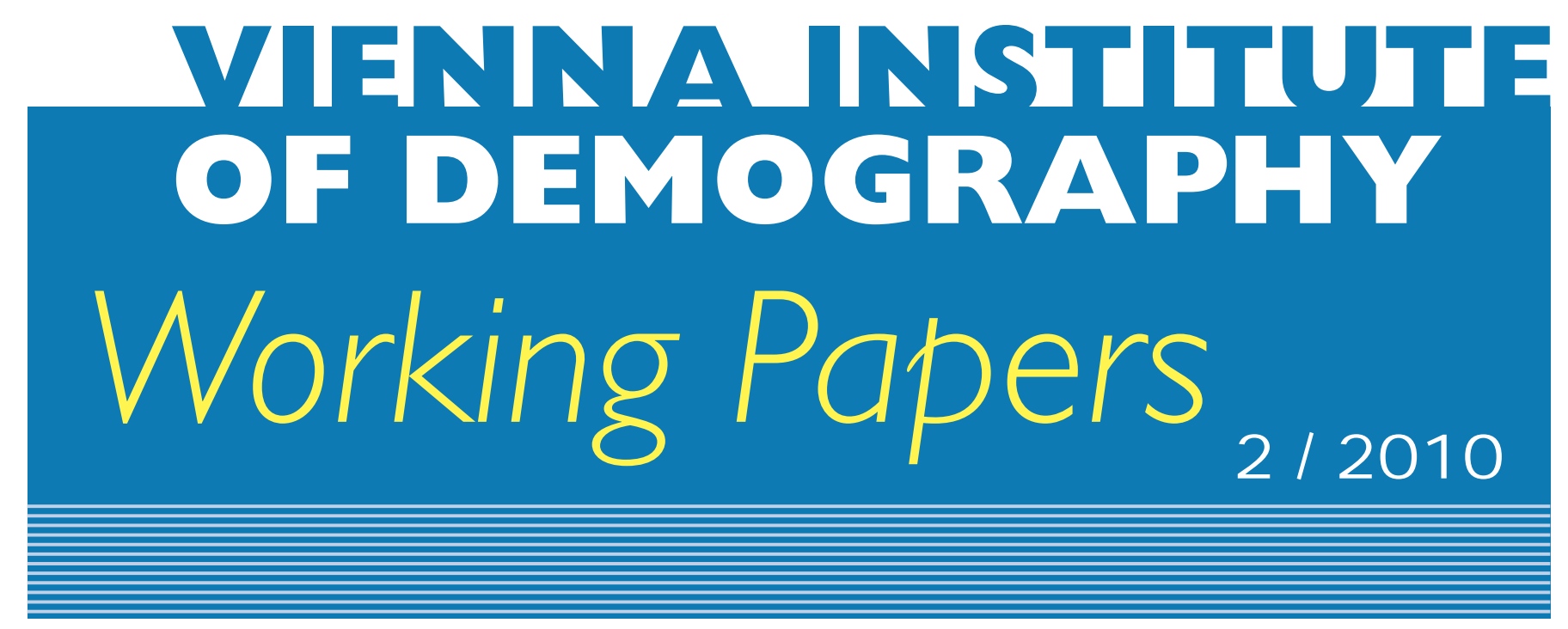

Isabella Buber

\title{
Wissenschaftlerinnen in Ö sterreich - Zusatzerhebung im Rahmen des GGS. Dokumentation der Datenerhebung und deskriptive Ergebnisse
}



Vienna Institute of Demography Austrian Academy of Sciences

Wohllebengasse $12-14$

A-I 040 Vienna - Austria

E-Mail:vid@oeaw.ac.at Website: www.oeaw.ac.at/vid

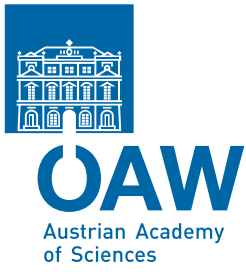




\begin{abstract}
The "Generations and Gender Survey (GGS)" is an important data source for studying the dynamics of families and family relationships, it was carried out in Austria in 2008/09. Additionally, the Austrian Academy of Sciences financed data collection among female scientists in Austria. In total, 247 women up to the age of 45 years who applied for a scholarship at the Austrian Academy of Sciences within the last ten years were interviewed the core GGS questionnaire as well as further questions related to scientific work. In this documentation, we inform about data collection, sample size and basic characteristics of the sample. Up to now, very little was known about the fertility behaviour of academic women in Austria, but also in other modern societies. The current sample - although very selective - will allow new insights in the fertility behaviour and in the family formation process of women working in the academic field.
\end{abstract}

\title{
Keywords
}

Generations and Gender Survey, female scientists, Austria, fertility, family

\section{Author}

Isabella Buber is research scholar at the Vienna Institute of Demography of the Austrian Academy of Sciences. Email: Isabella.Buber-Ennser@,oeaw.ac.at

\section{Acknowledgements}

I want to thank Caroline Berghammer for helpful comments as well as Heike Barakat for editing. 


\title{
Wissenschaftlerinnen in Österreich - Zusatzerhebung im Rahmen des GGS. \\ Dokumentation der Datenerhebung und deskriptive Ergebnisse
}

\author{
Isabella Buber
}

\section{GGS-Zusatzerhebung unter Wissenschaftlerinnen}

Im Zuge der Vorbereitungen für die Befragung „Generations and Gender Survey (GGS) 2008/09“ in Österreich entstand die Idee, im Rahmen einer Zusatzerhebung Wissenschaftlerinnen zu befragen, um mehr über ihre Pläne zur Gründung einer eigenen Familie, die Anzahl der gewünschten Kinder und ihre Ansichten zur Vereinbarkeit von Familie und Beruf sowie ihre Wünsche an die Familienpolitik zu erfahren. Die Österreichische Akademie der Wissenschaften (ÖAW) fördert mit einer Reihe von Stipendien den wissenschaftlichen Nachwuchs und hat darüber hinaus ein besonderes Interesse an der Verbesserung der Rahmenbedingungen ambitionierter Wissenschaftlerinnen.

Die Gruppe der Akademikerinnen ist in herkömmlichen Befragungen zumeist nur gering besetzt, sodass über Aspekte wie Familiengründung, Fertilität und berufliche Situation von Wissenschaftlerinnen in Österreich nur sehr wenig bekannt ist. Da der GGS für die österreichische Gesamtbevölkerung repräsentativ ist, sind unter den befragten Personen nur wenige Wissenschaftlerinnen. Aus diesem Grund hat sich die ÖAW entschlossen, eine zusätzliche Umfrage unter Wissenschaftlerinnen durchzuführen. Als Basis für die Zusatzerhebung diente die Adressendatei der Stipendienstelle der ÖAW.

So wurden - zusätzlich zu den bereits 5.000 interviewten Frauen und Männern - zwischen Juli und September 2009 insgesamt 247 Stipendienantragstellerinnen der Österreichischen Akademie der Wissenschaften befragt, um ein ausgewiesenes „WissenschaftlerinnenSample“ zur Verfügung zu haben. Die Finanzierung dieser Erhebung erfolgte aus Mitteln der Österreichischen Akademie der Wissenschaften.

Neben dem umfangreichen Fragenkatalog des international akkordierten GGS, konnten einige neue Fragen aufgenommen werden, die speziell für den wissenschaftlichen Bereich von Bedeutung sind (siehe Codebuch in Kapitel 8).

\section{Tätigkeitsbereich der ÖAW-Stipendienstelle}

Die ÖAW fördert mit einer Reihe von Stipendien den wissenschaftlichen Nachwuchs sowie hochqualifizierte WissenschaftlerInnen. Die Verwaltungsstelle für Stipendien und Preise entwickelt und verwaltet die Stipendienprogramme und vergibt Preise aus zweckgebundenen öffentlichen Mitteln, aus Drittmitteln und mit Unterstützung durch private SponsorInnen. 
Geleitet wird die Stipendienstelle von Dr. Barbara Haberl, der wir für die Möglichkeit der Realisierung unseres gemeinsamen Projekts, für ihre Unterstützung und die außerordentlich gute Zusammenarbeit danken möchten. Unser besonderer Dank gilt auch Frau Birgit Distler-Gamauf für ihre kompetente Hilfe.

Die Förderung exzellenter NachwuchswissenschaftlerInnen durch die Akademie schließt auch die Betreuung der StipendiatInnen ein. Dazu gehören die Organisation von Veranstaltungen zur Förderung intensiver Kommunikation und Vernetzung zwischen den jungen ForscherInnen sowie Angebote im Bereich Karriereberatung, die auch persönliche Coachings zur Ausbildung notwendiger Schlüsselqualifikationen umfassen. In dieser Hinsicht unterscheidet sich die ÖAW als Nachwuchsfördereinrichtung von allen anderen österreichischen Förderinstitutionen (ÖAW, 2008)

Das kontinuierlich wachsende Engagement der ÖAW im Bereich der Nachwuchsförderung begann im Jahr 1993 mit der Entwicklung des Programms APART, einer Förderschiene für WissenschaftlerInnen, die sich für eine Professur qualifizieren wollen. Derzeit schreibt die ÖAW sieben Stipendienprogramme aus - darunter drei Programme für DoktorandInnen mit unterschiedlichen Zielsetzungen - und vergibt 16 Forschungspreise in allen Fachbereichen. Im Jahr 2009 wurden mehr als 240 junge WissenschaftlerInnen gefördert.

\subsection{Angebotene Förderungen der ÖAW}

Hier eine Kurzbeschreibung der angebotenen Stipendien der ÖAW:

- APART (Austrian Programme for Advanced Research and Technology) Zielgruppe: junge, hoch qualifizierte WissenschaftlerInnen, die sich habilitieren oder eine habilitationsähnliche Leistung erbringen wollen, Projektdurchführung sowohl im In- als auch im Ausland Höchstalter 35 Jahre oder max. fünf Jahre seit Abschluss des Doktoratstudiums, österreichische Staatsbürgerschaft oder Durchführung des Forschungsprojektes an einer österreichischen Institution, Nachweis von Forschungserfahrung durch wissenschaftliche Publikationen.

Höhe des Stipendiums: 55.000,- Euro brutto jährlich

Dauer des Stipendiums: max. 36 Monate

- DOC (DoktorandInnenprogramm der Österreichischen Akademie der Wissenschaften)

Zielgruppe: hoch qualifizierte DissertantInnen

Höchstalter 27 Jahre oder max. 2 Jahre seit Abschluss des Diplom- oder Masterstudiums, Berechtigung für den Eintritt in ein Doktorats- oder PhD-Studium, österreichische Staatsbürgerschaft oder Durchführung der Dissertation an einer österreichischen Institution

Höhe des Stipendiums: 30.000,- Euro brutto jährlich

Dauer des Stipendiums: max. 36 Monate

- DOC-team (DoktorandInnengruppen für disziplinenübergreifende Arbeiten in den Geistes-, Sozial- und Kulturwissenschaften) 
Förderungsprogramm für Bewerbungen von DoktorandInnengruppen (3-5 Personen) aus den Geistes-, Sozial- und Kulturwissenschaften.

Zielgruppe: hoch qualifizierte DissertantInnen

Höchstalter 29 Jahre, Berechtigung für den Eintritt in ein Doktorats- oder PhDStudium, österreichische Staatsbürgerschaft oder Durchführung der Dissertation an einer österreichischen Institution

Höhe des Stipendiums: 30.000,- Euro brutto jährlich pro Person

Dauer des Stipendiums: max. 36 Monate

- DOC-fFORTE (Frauen in Forschung und Technologie)

Stipendium für junge Wissenschaftlerinnen aus den Bereichen Technik, Naturwissenschaften und Medizin sowie Biowissenschaften und Mathematik. ${ }^{1}$ Zielsetzungen der Förderinitiative: Durch DOC-fFORTE soll die Steigerung der Zweitabschlüsse (i.e. Doktorat, PhD) von Frauen in den genannten Disziplinen forciert werden. Dadurch soll die Präsenz von Frauen in leitenden bzw. verantwortlichen Positionen in technisch-naturwissenschaftlichen Berufen, in der außeruniversitären Forschung und im Unternehmenssektor erhöht werden.

Zielgruppe: hoch qualifizierte Doktorandinnen aus den Bereichen Technik und Naturwissenschaften sowie Biowissenschaften, Mathematik und Medizin

Höchstalter 27 Jahre oder max. 2 Jahre seit Abschluss des Diplom- oder Masterstudiums, Berechtigung für den Eintritt in ein Doktorats- oder PhD-Studium, österreichische Staatsbürgerschaft oder Durchführung der Dissertation an einer österreichischen Institution

Höhe des Stipendiums: 30.000,- Euro brutto jährlich

Dauer des Stipendiums: max. 36 Monate

- ROM (Stipendien am Historischen Institut beim Österreichischen Kulturforum in Rom)

Forschungsstipendien am Historischen Institut beim Österreichischen Kulturforum in Rom für graduierte oder promovierte AkademikerInnen.

Zielgruppe: hoch qualifizierte Wissenschaftlerinnen und Wissenschaftler aus den Bereichen der Geistes- und Sozialwissenschaften bzw. der Theologie

Höchstalter 29 Jahre (pre-doc) bzw. 34 Jahre (post-doc), abgeschlossenes Diplombzw. Masterstudium oder Doktorat, österreichische Staatsbürgerschaft oder Lebensmittelpunkt Österreich, ein konkretes Projekt mit thematischem Bezug zu Rom oder Italien, das einen Rom-Aufenthalt nötig macht

Höhe des Stipendiums: 900,- Euro brutto pro Monat

Dauer des Stipendiums: 1-9 Monate

Zusätzlich bietet die ÖAW derzeit folgende Stipendien an: AAS-CEE (Austrian Academy of Sciences Central and Eastern European Fellowship), FLARE (Future Leaders of Ageing Research in Europe), L'ORÉAL Österreich (Stipendien für junge GrundlagenForscherinnen in Österreich) und MAX KADE (USA-Stipendien der Max KadeFoundation). AAS-CEE wurde zum Zeitpunkt der Kontaktaufnahme zu den Frauen erst

\footnotetext{
${ }^{1}$ Finanziert vom Bundesministerium für Wissenschaft und Forschung (bmwf) im Rahmen des vom bmwf und dem Rat für Forschung und Technologieentwicklung initiierten Maßnahmenpakets "Frauen in Forschung und Technologie - fFORTE".
} 
einmal vergeben, der Stipendiat war darüber hinaus männlich. L'OREAL wurde ebenfalls bis zum Stichtag erst einmal vergeben, unter den StipendiatInnen befanden sich lediglich vier Frauen. MAX KADE AntragsstellerInnen wurden erst 1997 von der Stipendienstelle der ÖAW übernommen, sie waren zuvor einer anderen Abteilung zugeordnet, wodurch MAX KADE Anstragstellerinnen erst seit 1997 in der Datenbank der Stipendienstelle geführt werden. Weiters ist anzumerken, dass MAX KADE StipendiatInnen zumeist aus dem Blickfeld der ÖAW-Stipendienstelle kommen, da ihre Betreuung von den amerikanischen Universitäten übernommen wird. ${ }^{2}$ Die geringe Anzahl von Antragstellerinnen für AAS-CEE, FLARE und L'OREAL sowie das Fehlen einer vollständigen Adressendatei für MAX-KADE Stipendien und eine nicht weiter durchgeführte Aktualisierung dieser Adressen sind als Gründe anzuführen, warum keine der kontaktierten Frauen diese Stipendien beantragte oder bezogen hat. Die Befragung beschränkt sich somit auf fünf Programme, nämlich APART, DOC, DOC-team, DOCfForte und ROM.

\subsection{Kontaktaufnahme und Antwortrate}

Basierend auf der Datei der StipendienantragstellerInnen seit dem Jahr $1993^{3}$, wählte die Stipendienstelle in einem ersten Schritt Frauen aus, die 1964 oder später geboren wurden und somit bei der Befragung 45 Jahre oder jünger waren. Das Kriterium traf auf insgesamt 1561 Frauen zu ,die in weiterer Folge auch als Grundgesamtheit bezeichnet werden. Sie wurden postalisch und per E-Mail gebeten, an der Befragung teilzunehmen.

Die Stipendienstelle der ÖAW ist sehr um die Aktualisierung ihrer Adressdatenbank bemüht. Dies gelingt bei den erfolgreichen StipendienantragstellerInnen, d.h. bei den ehemaligen und aktuellen StipendiatInnen sehr gut, da es ein Anliegen der ÖAW ist, mit diesen Personen in Kontakt zu bleiben, um sie in vielfältiger Weise zu unterstützen. Die Aktualisierung der Adressen jener Personen, deren Antrag nicht positiv evaluiert werden konnte, gestaltet sich hingegen schwierig; zumeist gab es nach dem negativen Bescheid keine weiteren Kontakte zur Stipendienstelle.

Tabelle 1: Erreichbarkeit und Umfragebereitschaft der Frauen

\begin{tabular}{|l|l|l|l|l|}
\hline & Abs. & Rel. & Abs. & Rel. \\
\hline Zusage & 281 & $18,0 \%$ & 281 & $24,7 \%$ \\
\hline Absage & 3 & $0,2 \%$ & 3 & $0,3 \%$ \\
\hline im Ausland & 62 & $4,0 \%$ & & \\
\hline nicht erreicht & 360 & $23,1 \%$ & & \\
\hline keine Antwort & 855 & $54,8 \%$ & 855 & $75,1 \%$ \\
\hline Summe & 1,561 & $100,0 \%$ & 1,139 & $100,0 \%$ \\
\hline
\end{tabular}

\footnotetext{
${ }^{2}$ Wir möchten uns bei Frau Distler für die entsprechende Hintergrundinformation bedanken.

${ }^{3}$ Wie bereits weiter oben erwähnt, erfolgte eine Einschränkung auf APART, DOC, DOC-team, DOC-fForte und ROM, da die übrigen Stipendien zum Zeitpunkt der Befragung erst kurze Zeit angeboten wurden und somit nur wenige Anträge gestellt worden waren bzw. die entsprechende Adressendatei erst kurze Zeit in Betreuung der Stipendienstelle der ÖAW war.
} 
Im Juli 2008 versandte die Stipendienstelle der ÖAW an die bereits erwähnten 1561 Frauen einen Brief im Namen der Präsidenten der ÖAW mit der Bitte um Teilnahme an der Erhebung. Die Interviewzusagen bzw. -absagen erfolgten per E-Mail an eine eigens hierfür eingerichtete Adresse, auf welche sowohl die Stipendienstelle als auch das Institut für Demographie Zugriff hatte. Da zahlreiche Briefe aufgrund ungültiger Adressen nicht zugestellt werden konnten, versandte die Stipendienstelle in einem zweiten Schritt im November 2008 E-Mails an jene Frauen, mit denen bis dahin noch kein Kontakt zustande gekommen war. Es waren jedoch auch eine Reihe von E-Mail-Adressen der Adressdatenbank der Stipendienstelle im November 2008 nicht mehr gültig, sodass die Bitte um Teilnahme nicht alle Frauen erreichte.

Insgesamt 360 Frauen konnten mit Sicherheit nicht kontaktiert werden: Der an sie geschickte Brief wurde als unzustellbar retourniert und darüber hinaus lag keine gültige EMail-Adresse vor. 62 Frauen waren nachweislich im Ausland, und obwohl einige ihre Teilnahme an der Befragung zugesagt hatten, wurden sie aus zwei Gründen nicht interviewt. Einerseits fand die Befragung face-to-face statt, andererseits hätten bei Einbeziehung von Frauen, die sich im Ausland aufhielten, die gesellschaftlichen, gesetzlichen und familienpolitischen Bedingungen des entsprechenden Landes berücksichtigt werden müssen, was aufgrund der zu geringen Fallzahlen keine soliden Analysen erlaubt hätte.

Für die Berechnung der Antwortraten verblieben nach Abzug der nachweislich nicht kontaktierten und der sich nachweislich im Ausland befindlichen Frauen 1.139 Adressen. ${ }^{4}$ Nur wenige Frauen schickten eine E-Mail, um mitzuteilen, dass sie nicht an der Befragung teilnehmen möchten $(0,3 \%)$. Sehr groß war die Gruppe derer, die weder auf den Brief noch auf die E-Mail reagierten $(75,1 \%)$, unter ihnen befanden sich auch Frauen, die nicht teilnehmen wollten, aber keine Absage per E-Mail schickten. Vermutlich erreichten Brief oder E-Mail einen Teil dieser Frauen nicht, da beispielsweise eine nicht mehr aktuelle Adresse angeschrieben oder die E-Mail an eine nicht mehr abgerufene E-Mail-Adresse geschickt wurde. Auch ist anzunehmen, dass besonders bei jenen Frauen, deren Stipendienantrag von der ÖAW abgelehnt wurde, wenig Interesse bestand, für eine Befragung der ÖAW zur Verfügung zu stehen.

Insgesamt 281 Frauen, die im Zeitraum der Befragung in Österreich waren, erklärten sich bereit, teilzunehmen. Dies entspricht einer Rücklaufquote von 24,7\%. Die von den Frauen zur Verfügung gestellten Kontaktadressen wurden an Statistik Austria weitergeleitet. Geschulte Mitarbeiterinnen von Statistik Austria, die bereits für die österreichweite GGSErhebung eingesetzt worden waren, kontaktierten die Frauen und führten die Interviews durch. Statistik Austria war bemüht, die Frauen von weiblichen Interviewpersonen befragen zu lassen, so wie dies bereits im österreichweiten GGS gehandhabt worden war. Insgesamt 19 Adressen wurden an männliche Interviewer weitergegeben, da im entsprechenden Sprengel Statistik Austria keine GGS-geschulten Interviewerinnen zur Verfügung hatte.

\footnotetext{
${ }^{4}$ Die Bearbeitung der eingegangenen E-Mails und die Wartung der entsprechenden Adressendatei erfolgte durch Frau DI Petra Schmutz.
} 
Von den insgesamt 281 kontaktierten Frauen, deren Adressen an Statistik Austria weitergegeben wurden, konnten 247 interviewt werden. Es kam zu 12 neutralen Ausfällen, weil die zu Befragenden nicht an der angegebenen Adresse wohnten und 22 Frauen verweigerten das Interview, obwohl sie sich ursprünglich ihre Zustimmung gegeben hatten. Die Ausschöpfungsrate von Statistik Austria betrug, unter Berücksichtigung der neutralen Ausfälle, somit $92 \%$.

Bezogen auf die Grundgesamtheit aller 1.561 Adressen entsprechen die 247 durchgeführten Interviews einer Antwortrate von 16\%, bezogen auf die (nachweislich) kontaktierten Frauen ergibt sich eine Antwortrate von 22\% (Tabelle 2).

Sowohl die Auswahlkriterien im Hinblick auf die Adressen als auch die geringe Teilnahmebereitschaft zeigen, dass das vorliegende Sample eine sehr selektive Gruppe von Frauen darstellt. Obwohl die Antwortrate bedauerlich niedrig ist, muss betont werden, dass eine Umfrage von knapp 250 Wissenschaftlerinnen bzw. Frauen mit wissenschaftlichen Ambitionen einzigartig für Österreich ist. Weiters ist zu unterstreichen, dass dies die erste österreichische Befragung von Wissenschaftlerinnen im Zusammenhang mit Familienbildung ist.

Viele der internationalen Studien über WissenschaftlerInnen basieren auf sehr selektiven Samples. So analysierte Kemkes-Grottenthaler (2003) 196 weibliche Fakultätsmitglieder der Universität Mainz, arbeiteten Wolf-Wende und Ward (2006) in einer US-Studie mit Daten von 117 Assistenzprofessorinnen mit Kindern unter 5 Jahren oder publizierte Armenti (2004) eine Arbeit basierend auf Angaben von 19 Professorinnen an kanadischen Universitäten.

In Studien anderer Länder über Frauen in wissenschaftlichen Berufen sind die Antwortraten teilweise ähnlich niedrig, teilweise bedeutend höher. So veröffentlichten Romanin und Over (1993) eine Studie zu Frauen in akademischen Berufen in Australien. Die Antwortrate für ihre schriftliche Umfrage betrug 75\%. Eine US-amerikanische Studie unter Fakultätsmitgliedern erreichte mit 1.215 Befragten eine Antwortrate von 65\% (Fox, 2005) und eine Studie an der schwedischen Universität Uppsala erzielte bei einer schriftlichen Befragung unter Studenten eine Rücklaufquote von 67\% (Lampic et al., 2005). In Deutschland nahmen an einer Befragung unter weiblichen Fakultätsmitgliedern an der Universität Mainz 37\% teil (Kemkes-Grottenthaler, 2003), und eine OnlineBefragung unter deutschen WissenschaftlerInnen ergab 22\% als Rücklaufquote (Lind 2008) und erreichte damit einen ähnlich niedrigen Wert wie die vorliegende Studie in Österreich.

Schließlich ist eine Publikation basierend auf einer E-Mail-Befragung unter Hochschulabsolventen der US-Universität von Western Ontario zu nennen, für die von der Autorin eine unterste Grenze von 18\% für die Antwortrate angegeben wurde. (Van Anders, 2004). Für diese Studie erhielten alle eingeschriebenen graduierten Studenten einen Fragebogen per E-Mail. Die Autorin beschreibt, dass die Schwierigkeit zu eruieren, wie viele Personen tatsächlich den Fragebogen per E-Mail erhielten, auch problematisch für die Berechnung der Antwortquote war. 
Ähnlich verhält es sich mit der vorliegenden Befragung der Stipendiatinnen der ÖAW. Die angegebene Antwortrate von 22\% ist als untere Grenze zu sehen, da möglicherweise ein erheblicher Teil der Frauen nicht erreicht wurde, weil einige der angeschriebenen Adressen nicht gültig waren und die entsprechenden Briefe nicht retourniert wurden, weil viele EMails mit Bitte um Teilnahme an E-Mail-Adressen versandt wurden, die nicht mehr abgerufen werden, oder weil die E-Mails aufgrund eines Filters in die Rubrik ,junk mails“ gelangten und deshalb nicht gelesen wurden.

Tabelle 2: Antwortrate

\begin{tabular}{|l|l|}
\hline Grundgesamtheit & 1.561 \\
\hline Kontaktierte & 1.139 \\
\hline Interviewte & 247 \\
\hline Anteil der Interviewten bezogen auf die Grundgesamtheit & $16 \%$ \\
\hline Anteil der Interviewten bezogen auf die kontaktierten Frauen & $22 \%$ \\
\hline
\end{tabular}

\subsection{Antwortbereitschaft}

Die Zusage bzw. Ablehnung des Stipendienantrags ist eine wesentliche Determinante sowohl für die Erreichbarkeit als auch für die Antwortbereitschaft. Erwartungsgemäß wurden Frauen mit abgelehntem Antrag öfter nicht erreicht (26\%) als Altstipendiatinnen (19\%) oder aktuelle Stipendiatinnen (2\%) (Tabelle 3). Dies lässt sich dadurch erklären, dass die Adressen der aktuellen Stipendiatinnen und der Altstipendiatinnen von der ÖAW aktualisiert werden, während es zu den abgelehnten Antragstellerinnen meist keine weiteren Kontakte gab und somit die entsprechenden Adressen nicht à jour gehalten werden konnten.

Beschränkt man sich auf die 1.139 nachweislich kontaktierten Frauen, so sind die Unterschiede bezüglich der Teilnahmebereitschaft noch deutlicher. Während sich die Hälfte der aktuellen Stipendiatinnen und vier von zehn Altstipendiatinnen für ein Interview bereit erklärten, konnten nur 18\% der erfolglosen Antragsstellerinnen für die Befragung gewonnen werden (Abbildung 1). Die Aussendung mit Bitte um Teilnahme an der Befragung erfolgte im Namen des Präsidenten der ÖAW. Die Bereitschaft zur Teilnahme war deutlich höher unter Frauen, die ein ÖAW-Stipendium erhalten hatten, was als Indiz dafür gesehen werden kann, dass sie sich der ÖAW zu einem gewissen Grad verbunden fühlen und eher bereit sind, sich für eine Befragung im Auftrag der ÖAW zur Verfügung zu stellen.

Tabelle 3: Erreichbarkeit der Frauen nach dem Status des Stipendienantrags

\begin{tabular}{|l|l|l|l|l|l|l|l|}
\hline & Zusage & Absage & Ausland & $\begin{array}{l}\text { nicht } \\
\text { erreicht }\end{array}$ & $\begin{array}{l}\text { keine } \\
\text { Antwort }\end{array}$ & Total & Abs, \\
\hline Altstipendiatin & $27 \%$ & $1 \%$ & $8 \%$ & $19 \%$ & $45 \%$ & $100 \%$ & 303 \\
\hline Antragstellerin & $13 \%$ & $0 \%$ & $3 \%$ & $26 \%$ & $58 \%$ & $100 \%$ & 1.162 \\
\hline Stipendiatin & $48 \%$ & $0 \%$ & $4 \%$ & $2 \%$ & $46 \%$ & $100 \%$ & 96 \\
\hline Total & $18 \%$ & $0 \%$ & $4 \%$ & $23 \%$ & $55 \%$ & $100 \%$ & 1.561 \\
\hline
\end{tabular}


Abbildung 1: Antwortbereitschaft unter den nachweislich kontaktierten Frauen, nach Status des Stipendienantrags

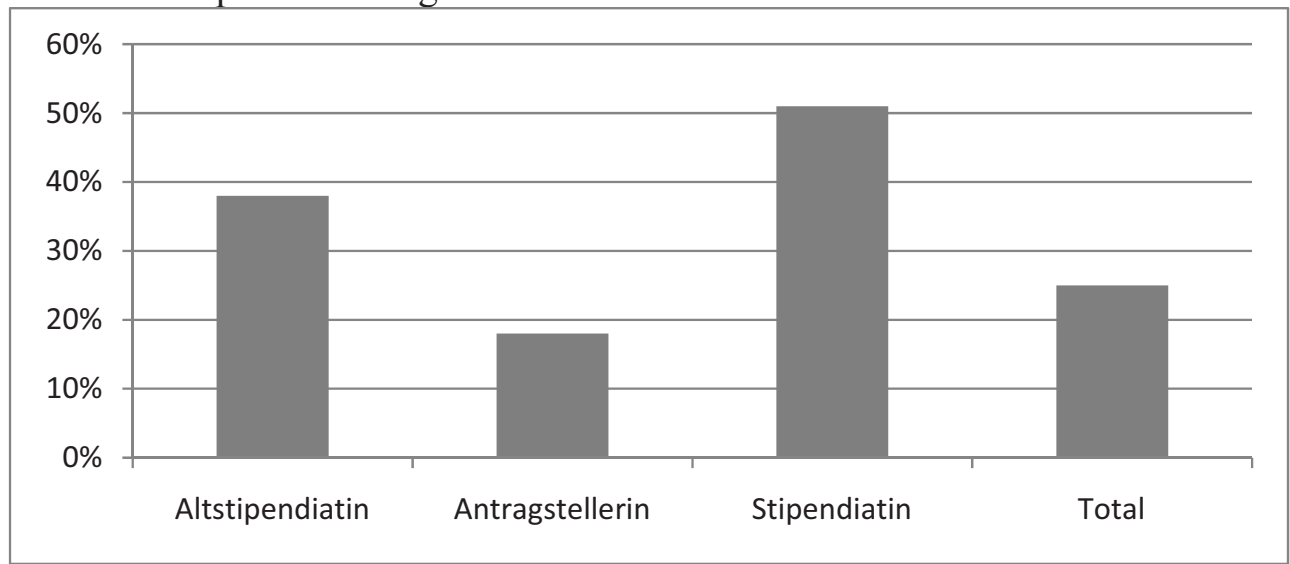

Bemerkung: $\mathrm{N}=1.139$ nachweislich kontaktierte Frauen

Es ist grundsätzlich zu betonen, dass das vorliegende Projekt nicht als Studie über die Förderungsprogramme der ÖAW angelegt ist, sondern generell auf Wissenschaftlerinnen fokussiert bzw. auf Frauen, die im Laufe ihrer Ausbildung und Berufstätigkeit eine wissenschaftliche Position anstrebten.

\subsection{Vergleich der befragten Frauen mit der Grundgesamtheit}

Deutlich zeigt sich eine Beziehung zwischen Antragsjahr und erfolgreich durchgeführtem Interview: Je länger der Antrag zurückliegt, desto geringer die Interviewrate. Von den Antragstellerinnen der 1990er Jahre konnten nur 9\% befragt werden, unter Frauen mit Anträgen aus den Jahren 2000 bis 2004 erhöht sich der Anteil auf 14\% und von den Antragstellerinnen aus den Jahren 2005 bis 2008 wurden 23\% interviewt (Tabelle 4). Bezogen auf die (nachweislich) kontaktierten Frauen erhöhen sich die Antwortraten auf 12\% (Antrag 1993-1999), 19\% (Antrag 2000-2004) und 33\% (Antrag 2005-2008).

Ein Vergleich der Altersverteilung in der Grundgesamtheit, der nachweislich kontaktierten Frauen und der tatsächlich Befragten, zeigt, dass unter den interviewten Frauen jüngere Geburtenkohorten (1980 bis 1984) eher überrepräsentiert, die Kohorten 1970 bis 1974 eher unterrepräsentiert sind (Abbildung 2). Fehlende aktuelle Adressen sind mit als Grund für die Unterrepräsentativität älterer Jahrgänge zu nennen. Es ist allerdings anzumerken, dass sich für Frauen der ältesten Geburtenkohorten (1964 bis 1969) kaum Unterschiede zeigen und diese ähnlich wie in der Grundgesamtheit auch unter den Interviewten vertreten sind. Dies ist insofern wichtig, da diese Frauen zum Zeitpunkt der Befragung 40 Jahre und älter waren, sich somit am Ende ihrer reproduktiven Phase befanden und damit Analysen zu ihrer (endgültigen) Kinderzahl möglich sind. Der vergleichsweise hohe Anteil interviewter Frauen der Geburtenjahrgänge 1980 bis 1984 lässt sich durch die hohe Antwortbereitschaft unter den aktuellen Stipendiatinnen erklären, von denen über die Hälfte diesen Geburtskohorten angehört. Diese Frauen waren zum Zeitpunkt der Befragung zwischen 25 und 29 Jahre alt. 
Tabelle 4: Antwortverhalten nach Jahr des Stipendienantrags

\begin{tabular}{|l|l|l|l|l|}
\hline & Gesamt & $1993-1999$ & $2000-2004$ & $2005-2008$ \\
\hline Grundgesamtheit & 1.560 & 418 & 626 & 516 \\
\hline Kontaktierte & 1.138 & 308 & 459 & 371 \\
\hline Interviewte & 247 & 38 & 88 & 121 \\
\hline $\begin{array}{l}\text { Anteil der Interviewten bezogen auf die } \\
\text { Grundgesamtheit }\end{array}$ & $16 \%$ & $9 \%$ & $14 \%$ & $23 \%$ \\
\hline $\begin{array}{l}\text { Anteil der Interviewten bezogen auf die } \\
\text { kontaktierten Frauen }\end{array}$ & $22 \%$ & $12 \%$ & $19 \%$ & $33 \%$ \\
\hline
\end{tabular}

Abbildung 2: Altersverteilung in der Grundgesamtheit, unter den nachweislich kontaktierten und den tatsächlich befragten Frauen

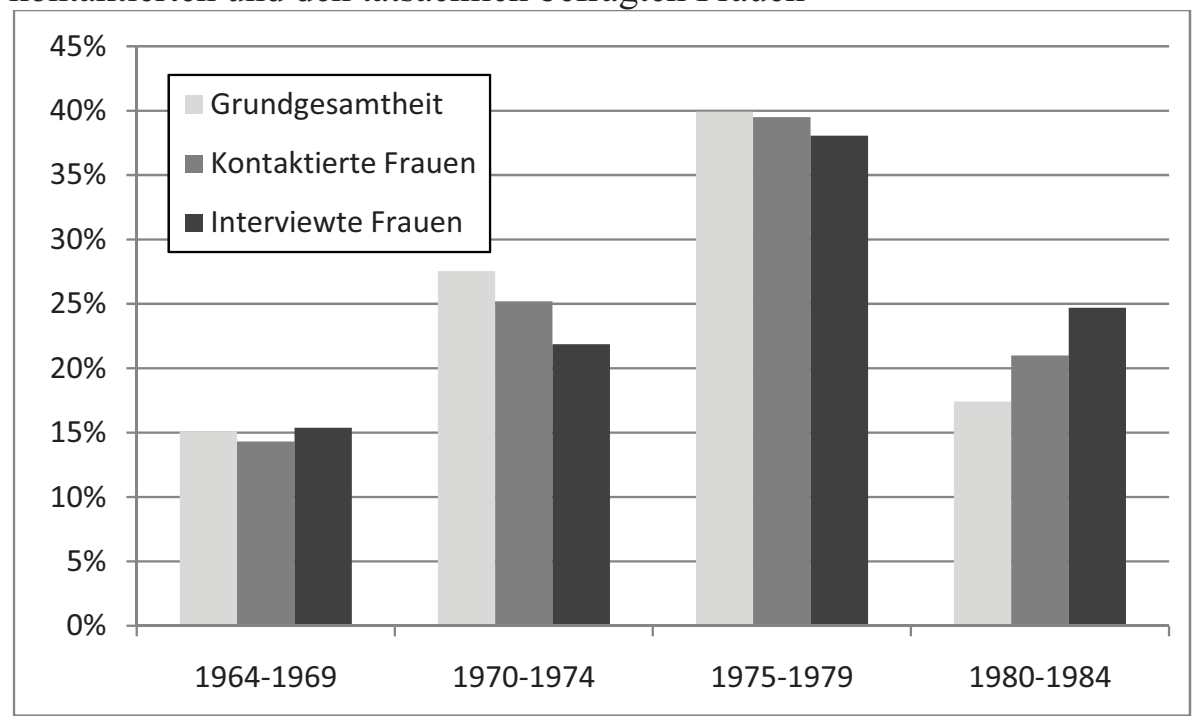

Grundgesamtheit: 1.561 Adressen; Kontaktiert: 1.139 Frauen; Befragt: 247 Frauen

Bezogen auf die beantragten Förderungen sind DOC-Stipendienantragstellerinnen unterrepräsentiert. Ein hoher Anteil von ihnen konnte wegen ungültiger Adressen nicht erreicht werden, und vergleichsweise wenige der nachweislich Kontaktierten stellten sich für ein Interview zur Verfügung (20\%) (siehe Tabellen A1 und A2 im Anhang). Es ist zu beachten, dass knapp 1.000 Frauen DOC-Antragstellerinnen waren und mit $60 \%$ die Mehrheit der kontaktierten Frauendarstellen. In der kleinen Gruppe der DOC-TEAMAntragstellerinnen hingegen war die Antwortbereitschaft vergleichsweise hoch (50\%).

Wie bereits erwähnt, ist eine positive Beurteilung des Stipendienantrags wesentlich für die Antwortbereitschaft. Die Erfolgsquote bei den Stipendienanträgen in der vorliegenden Grundgesamtheit beträgt 26\%, sie ist aber für die einzelnen Stipendien unterschiedlich und bei DOC mit 22\% vergleichsweise niedrig, bei DOC-TEAM relativ hoch (55\%). Es ist aber zu beachten, dass die vorliegenden Angaben nicht mit den allgemeinen Erfolgsquoten der ÖAW-Stipendienanträge übereinstimmen, da hier ein spezielles Sample gezogen wurde 
(Frauen, die 1964 oder später geboren wurden). Während in der Grundgesamtheit (1.561 Frauen) ein Viertel ein Stipendium der ÖAW erhalten hat, beträgt die Erfolgsquote bei den interviewten Frauen 47\% (Abbildung 3). Ob die Frauen an anderen Stellen, etwa beim Fonds zur Förderung der wissenschaftlichen Förderung (FWF) erfolgreich waren, geht aus den Daten nicht hervor.

Abbildung 3: Anteil der Stipendiatinnen und Altstipendiatinnen in der Grundgesamtheit, bei den kontaktierten Frauen und bei den interviewten Frauen, nach Stipendienart

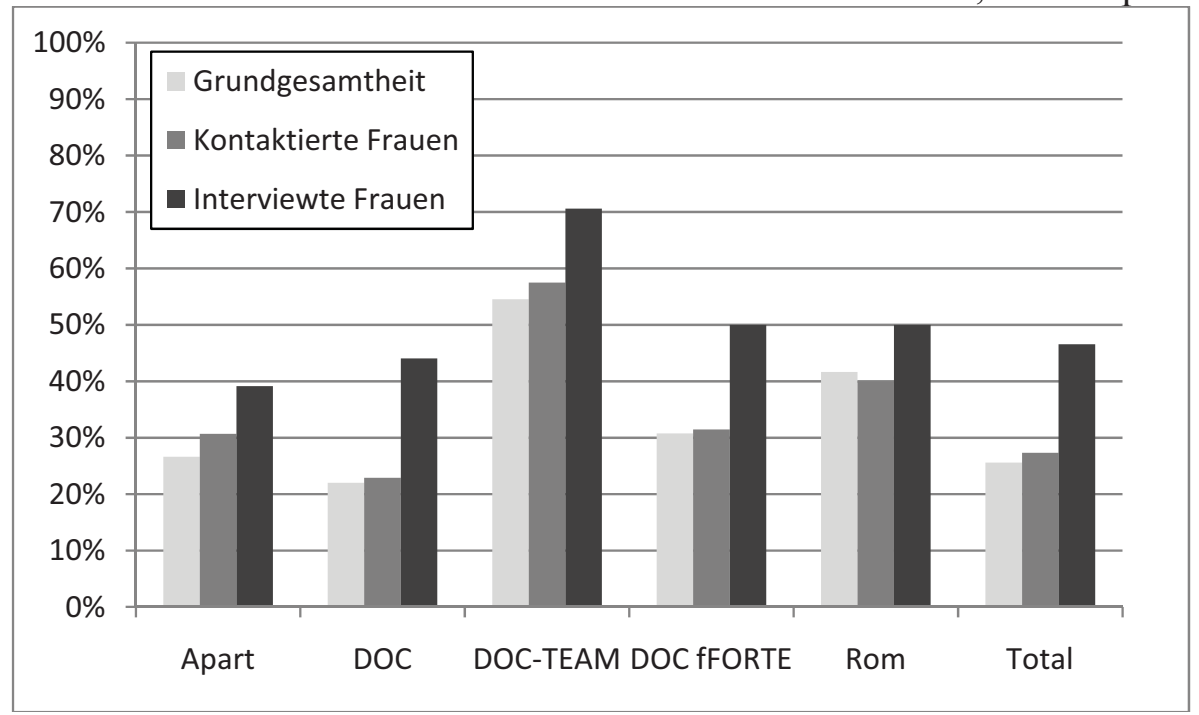

Grundgesamtheit: 1.561 Adressen; Kontaktiert: 1.139 Frauen; Befragt: 247 Frauen

\section{Deskriptive Ergebnisse zu den Wissenschaftlerinnen}

Das Ziel der ersten Phase der Datenanalyse bestand darin, einen Überblick über das vorliegende Datenmaterial zu erhalten. Folgende Fragen waren hierfür von Interesse: Welche Frauen konnten befragt werden? Erklären sich eher erfolgreiche Stipendienantragstellerinnen bereit, an der Befragung teilzunehmen? Um welche Stipendien haben sich die befragten Frauen beworben? Wie lange liegt der Stipendienantrag zurück? Wie viele Jahre sind bei den erfolgreichen Antragstellerinnen seit dem Ende ihres Stipendiums vergangen? Wie ist ihre fachliche Ausrichtung? Diese Informationen sind wichtig, um zu sehen, in welche Richtungen weitergeforscht werden kann, und für welche Fragestellungen zu wenige Datensätze vorhanden sind.

\subsection{Stipendienbewilligung und zur Stipendienart}

Von den fünf betrachteten Stipendienprogrammen sind drei für DoktorandInnen (DOC, DOC-team und DOC-fFORTE) und eines für promovierte AkademikerInnen im Zuge ihrer Habilitationsarbeit (Apart). Rom-Stipendien sind am Historischen Institut beim Österreichischen Kulturforum in Rom angesiedelt und für promovierte und graduierte AkademikerInnen vorgesehen. Anträge für dieses spezielle Stipendium sind zahlenmäßig gering. 
Insgesamt 218 der 247 befragten Frauen haben einmal um ein ÖAW-Stipendium angesucht. 29 Frauen haben zweimal oder öfter bei der ÖAW ein Stipendium beantragt. ${ }^{5}$ $\mathrm{Ob}$ auch andere Stipendien beantragt wurden, etwa beim Fonds zur Förderung der wissenschaftlichen Forschung (FWF), ist nicht bekannt. Es ist aber anzunehmen, dass dies durchaus geschah. Im Falle mehrerer Stipendienanträge konzentrieren wir uns auf das (höchste) zuerkannte Stipendium bzw. auf das höchste beantragte Stipendium (Apart).

DOC-Antragstellerinnen machen die Hälfte aller befragten Frauen aus. In Summe haben sich drei Viertel der Frauen um ein Doktoranden-Programm beworben, ein Viertel um ein Stipendium für promovierte AkademikerInnen (Tabelle 5).

Von Seiten der ÖAW gibt es Informationen zum Status der Stipendienanträge, der zwischen Altstipendiatin, Antragstellerin und Stipendiatin unterscheidet. Eine Altstipendiatin ist eine Frau, deren Stipendium positiv beurteilt wurde und die dieses sowohl angetreten als auch beendet hat. ${ }^{6}$ Eine Stipendiatin ist eine Frau, die aktuell ein Stipendium der ÖAW bezieht, und schließlich gibt es die Antragstellerinnen, deren Antrag aus verschiedenen Gründen nicht erfolgreich war, oder die auf einer Warteliste stehen.

Insgesamt 115 und somit 47\% der Befragten haben ein Stipendium der ÖAW erhalten (31\%: Stipendium abgeschlossen, 16\% aktuell Stipendiatin), 54\% der Befragten hatten ohne Erfolg einen oder mehrere Anträge bei der ÖAW gestellt. Es liegen damit zwei etwa gleich große Gruppen von Frauen vor, die ein Stipendium erhalten bzw. nicht erhalten haben und verglichen werden können.

Tabelle 5: Beantragtes Stipendienprogramm

\begin{tabular}{|l|l|l|l|l|}
\hline & \multicolumn{2}{|l|}{$\begin{array}{l}\text { Alle befragte } \\
\text { Frauen }\end{array}$} & \multicolumn{2}{l|}{$\begin{array}{l}\text { (Alt-) } \\
\text { Stipendiatinnen }\end{array}$} \\
\hline & Abs, & Rel, & Abs, & Rel, \\
\hline Apart & 46 & $21 \%$ & 18 & $16 \%$ \\
\hline DOC & 118 & $50 \%$ & 52 & $45 \%$ \\
\hline DOC-TEAM & 17 & $4 \%$ & 12 & $10 \%$ \\
\hline DOC-fFORTE & 58 & $22 \%$ & 29 & $25 \%$ \\
\hline Rom & 8 & $3 \%$ & 4 & $3 \%$ \\
\hline Total & 247 & $100 \%$ & 115 & $100 \%$ \\
\hline
\end{tabular}

Die 115 (Alt-)Stipendiatinnen bezogen in der Mehrzahl Doktorandenprogramme (DOC: 45\%; DOC-team: 10\%; DOC fFORTE 25\%), aber auch Habilitandinnen sind unter den befragten Frauen (APART: 16\%; Rom: 3\%).

\footnotetext{
${ }^{5}$ Eine Frau hat vier Anträge bei der ÖAW eingebracht. Eine einzige Frau im Datensatz zwei Stipendien von der ÖAW erhalten (DOC und APART), in diesem Fall konzentrieren wir uns auf das höchste (APART). Alle übrigen haben ein oder kein ÖAW-Stipendium bekommen.

${ }^{6}$ Insgesamt 7 Frauen im Datensatz haben ihr Stipendium abgebrochen. Laut Rückfrage bei der Stipendienstelle sind Gründe dafür zumeist interessante Stellenangebote, in einem Fall ging eine Frau in Elternkarenz.
} 


\subsection{Zeitpunkt des Stipendienantrags}

Das Jahr des Stipendienantrags sowie Beginn und Ende der angetretenen Stipendien sind bekannt (vgl. Tabelle A3). Informationen darüber, ob der Stipendiumsantrag bzw. das absolvierte Stipendium schon länger zurückliegt, oder erst vor wenigen Jahren erfolgte, sind wichtig für die Analyse der Daten. Unter den tatsächlich befragten Frauen stammt der früheste Stipendienantrag aus dem Jahr 1993, der jüngste aus dem Jahr 2007. Die ersten Stipendien wurden 1993 und 1995 begonnen, die letzten 2008. Die ersten Stipendien wurden 1996 abgeschlossen, die zum Zeitpunkt der Befragung laufenden Stipendien werden bis 2009 bzw. 2010 ausbezahlt.

Tabelle 6: Jahr des Stipendienantrags

\begin{tabular}{|l|l|l|}
\hline & Abs. & Rel. \\
\hline $1993-1999$ & 38 & $15 \%$ \\
\hline $2000-2004$ & 88 & $36 \%$ \\
\hline $2005-2007$ & 121 & $49 \%$ \\
\hline Total & 247 & $100 \%$ \\
\hline
\end{tabular}

Nur wenige Antragstellerinnen und Stipendiatinnen aus den 1990er Jahren konnten interviewt werden, nur 15\% der Anträge wurden vor dem Jahr 2000 eingereicht. Dies hängt damit zusammen, dass ein Teil der Antragstellerinnen der 1990er Jahre zum Stichtag älter als 45 Jahre war und somit nicht mehr als Interviewpartnerinnen in Frage kam.

Die Hälfte der Stipendien wurde 2005 oder später angetreten, insgesamt 39 Frauen bezogen zum Zeitpunkt der Befragung noch das ÖAW Stipendium. Dies bedeutet für Analysen basierend auf dem vorliegenden Datensatz, dass in den meisten Fällen erst wenige Jahre seit dem Ende des Stipendiums vergangen sind, und sich die meisten Frauen am Beginn oder in der ersten Phase ihrer wissenschaftlichen Karriere befinden.

\subsection{Fachliche Ausrichtung}

Unter den 247 befragten Frauen haben 245 einen Universitätsabschluss, zwei einen Fachhochschulabschluss. Die Mehrzahl der befragten Frauen hat ein abgeschlossenes Doktorat (57\%).

Eine wichtige Information stellt die exakte Bezeichnung der Studienrichtung sowie die generelle fachliche Ausrichtung dar. Dazu wurde das Ausbildungsfeld bzw. die Fachrichtung der höchsten abgeschlossenen Ausbildung (Eurostat 1999) codiert. Nachfolgende Tabelle 7 enthält jene fachlichen Ausrichtungen, die im Wissenschaftlerinnen-Sample vertreten sind. 
Tabelle 7: Hauptgegenstand der Ausbildung

\begin{tabular}{|c|c|}
\hline \multicolumn{2}{|c|}{ Allgemeine Bildungsgänge } \\
\hline & Erstdurchforstung \\
\hline \multicolumn{2}{|c|}{ Pädagogik } \\
\hline & $\begin{array}{l}\text { Biologie und Umweltkunde - Lehramt; Lehramt; Mathematik, DG, Physik, Chemie - } \\
\text { Lehramt; Physik - Lehramt; Sportwissenschaften u. Leibeserziehung; Werkerziehung - } \\
\text { Lehramt }\end{array}$ \\
\hline & Erziehungswissenschaft; Pädagogik \\
\hline \multicolumn{2}{|c|}{ Geisteswissenschaften und Künste } \\
\hline & Kunstgeschichte \\
\hline & Konservierung und Restaurierung; Musik; Musikwissenschaft; Theaterwissenschaft \\
\hline & Graphik-Design; Medienmanagement \\
\hline & Theologie \\
\hline & $\begin{array}{l}\text { Anglistik; Französisch; Germanistik; } \\
\text { Sprachwissenschaft; Ägyptologie }\end{array}$ \\
\hline & $\begin{array}{l}\text { Alte Geschichte und Altertumskunde; Archäologie; Philosophie; Geschichte; Klassische } \\
\text { Archäologie; Ur- und Frühgeschichte; Vergleichende Literaturwissenschaft }\end{array}$ \\
\hline \multicolumn{2}{|r|}{ Sozialwissenschaften, Wirtschafts- und Rechtswissenschaften } \\
\hline & $\begin{array}{l}\text { Demographie; Ethnologie; Politikwissenschaften; Psychologie; Sozialwissenschaften; } \\
\text { Soziologie; Volkswirtschaft(slehre) }\end{array}$ \\
\hline & Kommunikationswissenschaften; Publizistik \\
\hline & Betriebswirtschaft; Handelswissenschaft; Internationale Wirtschaftswissenschaften \\
\hline & Jura; Rechtswissenschaft \\
\hline \multicolumn{2}{|c|}{ Naturwissenschaften, Mathematik und Informatik } \\
\hline & Biochemie; Biologie; Mikrobiologie; Molekularbiologie; Zoologie \\
\hline & Astronomie; Biochemie; Biologie; Chemie; Geographie; Geologie; Physik \\
\hline & Mathematik; Technische Mathematik \\
\hline & Informatik \\
\hline & Naturwissenschaften \\
\hline \multicolumn{2}{|c|}{ Ingenieurwesen, Herstellung und Baugewerbe } \\
\hline & Telematik \\
\hline & Technische Chemie; Verfahrenstechnik \\
\hline & Ernährungswissenschaft; Lebensmittel- und Biotechnologie \\
\hline & Architektur; Landschaftspflege und Landschaftsplanung \\
\hline & Bauingenieurwesen \\
\hline \multicolumn{2}{|c|}{ Agrarwissenschaft und Veterinärwissenschaft } \\
\hline & Landwirtschaft \\
\hline & Veterinärmedizin \\
\hline \multicolumn{2}{|c|}{ Gesundheit und soziale Dienste } \\
\hline & Medizin \\
\hline & Erste Hilfe; Pharmazie \\
\hline & Sozialpolitik \\
\hline \multicolumn{2}{|c|}{ Dienstleistungen } \\
\hline & Umweltwissenschaften; Ökologie \\
\hline
\end{tabular}

Bemerkung: Die Auflistung enthält die sogenannten breiten Felder der ISCED-97 Klassifizierung sowie die sogenannten detaillierten Felder (dreistellig codierte Ausbildungsfelder) (Eurostat 1999).

Werden die exakten Bezeichnungen der Studienrichtungen zusammengefasst, so ergibt sich folgende Gruppierung (Tabelle 8): Ein Drittel der befragten Frauen absolvierte ein geisteswissenschaftliches Studium oder ein Kunststudium, zwei von zehn haben einen 
Abschluss in einem sozialwissenschaftlichen Fach, in Wirtschaft oder in Recht, 28\% der befragten Frauen haben ein naturwissenschaftliches Studium abgeschlossen. Im Sample sind nur wenige Absolventinnen aus den Fächern Erziehung, Gesundheit, Landwirtschaft oder Dienstleistungen vertreten. In den einzelnen Studienrichtungen liegt der Anteil der Frauen mit abgeschlossenem Doktorat zwischen $45 \%$ und $75 \%$. Es ist auch hier wieder zu beachten, dass die befragten Frauen nicht repräsentativ für die Absolventinnen ihrer Studienrichtungen sind.

Tabelle 8: Studienrichtung

\begin{tabular}{|l|l|l|l|}
\hline & Abs. & Rel. & Anteil mit Doktorat \\
\hline Pädagogik & 10 & $4 \%$ & $50 \%$ \\
\hline Geisteswissenschaften und Künste & 79 & $32 \%$ & $65 \%$ \\
\hline $\begin{array}{l}\text { Sozialwissenschaften, Wirtschafts- und } \\
\text { Rechtswissenschaften }\end{array}$ & 53 & $21 \%$ & $47 \%$ \\
\hline Naturwissenschaften, Mathematik und Informatik & 69 & $28 \%$ & $59 \%$ \\
\hline Ingenieurwesen, Herstellung und Baugewerbe & 20 & $8 \%$ & $45 \%$ \\
\hline Agrarwissenschaft und Veterinärwissenschaft & 3 & $1 \%$ & $67 \%$ \\
\hline Gesundheit und soziale Dienste & 9 & $4 \%$ & $67 \%$ \\
\hline Dienstleistungen & 4 & $2 \%$ & $75 \%$ \\
\hline Total & 247 & $100 \%$ & $57 \%$ \\
\hline
\end{tabular}

Vier von zehn befragten Wissenschaftlerinnen befanden sich zum Zeitpunkt der Befragung in einer Ausbildung. Bei dieser Ausbildung dürfte es sich vermutlich um das Doktorat gehandelt haben, da $87 \%$ der in Ausbildung befindlichen Frauen keinen Doktortitel angegeben hatten. Schließlich planten $20 \%$ der befragten Wissenschaftlerinnen in den nächsten drei Jahren eine weitere Ausbildung. Diese geplante Ausbildung hat vermutlich Auswirkungen auf den unmittelbaren und mittelfristigen Kinderwunsch der Frauen.

Im GGS wurde nicht nur eine Ausbildung abgefragt, es bestand auch die Möglichkeit, weitere abgeschlossene Ausbildungen anzugeben. 36\% der befragten Frauen gaben an, eine weitere Ausbildung abgeschlossen zu haben. Genannt wurden MBA, MAS und andere postgraduate Lehrgänge, aber auch nicht-akademische Ausbildungen (wie z.B.: Heilmasseurin oder Coaching).

\subsection{Beschäftigung}

Angaben zum aktuellen Beschäftigungsstatus sind wesentliche Informationen, die auch Bestandteil des internationalen GGS-Fragebogens sind. Der Großteil (89\%) der befragten Wissenschaftlerinnen war zum Zeitpunkt der Befragung beschäftigt, einige Frauen befanden sich in Mutterschutz oder Karenz (Tabelle 9).

Nur sehr wenige waren arbeitslos, Hausfrauen oder Studentinnen. Diese Gruppen sind nicht repräsentativ vertreten und können daher mit dem vorliegenden Datenmaterial nicht speziell analysiert werden. Es können aufgrund des Anteils der erwerbstätigen Interviewten keine allgemeinen Angaben zur Beschäftigungssituation der Wissenschaftlerinnen in Österreich gemacht werden, da das vorliegende Sample zu 
speziell ist. ${ }^{7}$ Von den 13 Frauen, die als Beschäftigungsstatus „Mutterschutz oder Karenz“ angegeben hatten, waren zwei in Mutterschutz, zehn in Karenz und in über die Karenzzeit hinausgehender Kinderbetreuungszeit. Auch die geringe Besetzung dieser Gruppe lässt keine weiteren Analysen der Wissenschaftlerinnen in Elternkarenz zu.

Tabelle 9: Aktueller Beschäftigungsstatus

\begin{tabular}{|l|l|l|}
\hline & Abs & In \% \\
\hline Angestellt oder selbständig & 219 & 89 \\
\hline Arbeitslos & 6 & 2 \\
\hline Student, Schüler, in Ausbildung & 7 & 3 \\
\hline Mutterschutz oder Karenz & 13 & 5 \\
\hline Hausfrau/Hausmann & 1 & 0 \\
\hline Sonstiges & 1 & 0 \\
\hline Total & 247 & 100 \\
\hline
\end{tabular}

\subsubsection{Tätigkeitsbereiche}

Die exakte Bezeichnung der aktuellen bzw. der letzten hauptberuflichen Tätigkeit wurde erfragt und sehr detailliert als vierstelliger ISCO-88-Bezeichnung codiert (Tabellen A5 und A6). Eine grobe Zusammenstellung zeigt Tabelle 10. Der Großteil der befragten Frauen war in akademischen Berufen tätig.

Tabelle 10: Bezeichnung der hauptberuflichen Tätigkeit

\begin{tabular}{|l|l|}
\hline & Abs. \\
\hline Führungskräfte & 15 \\
\hline Akademische Berufe & 191 \\
\hline Techniker und gleichrangige nichttechnische Berufe & 25 \\
\hline Bürokräfte und verwandte Berufe & 8 \\
\hline Dienstleistungsberufe, Verkäufer & 2 \\
\hline Fachkräfte in der Land- und Forstwirtschaft sowie Fischerei & 1 \\
\hline Total & 242 \\
\hline $\begin{array}{l}\text { Bemerkung: einstellige Zusammenfassung der ISCO-88-Codierung der aktuellen bzw. der letzten } \\
\text { Beschäftigung }\end{array}$
\end{tabular}

Die exakten Berufsbezeichnungen nach ISCO-88 sowie die weiter unten beschriebenen Angaben zum Tätigkeitsbereich ermöglichen es, die tatsächlich in wissenschaftlichen Bereichen tätigen Frauen zu selektieren und zu analysieren. Wie zu Beginn erwähnt, wurden Frauen kontaktiert, die sich um ein Stipendium der ÖAW beworben hatten, die also $\mathrm{zu}$ einem bestimmten Zeitpunkt Ambitionen für eine wissenschaftliche Tätigkeitzeigten. Nicht alle Frauen realisierten dieses Vorhaben, einige wechselten in andere Tätigkeitsbereiche. Die vorliegenden detaillierten Angaben zur Beschäftigung ermöglichen es künftigen Studien Frauen zu identifizieren, die in Wissenschaft und Forschung tätig sind.

\footnotetext{
${ }^{7}$ Unter den 3.000 befragten Frauen im Rahmen der österreichweiten Erhebung war die Beschäftigungsrate unter den gleichaltrigen Akademikerinnen und Nicht-Akademikerinnen mit 81\% bzw. 79\% niedriger.
} 


\subsubsection{Beschäftigungsumfang, Arbeitsort, Jobdauer}

Durchschnittliche Arbeitsstunden und das Beschäftigungsausmaß sind wie folgt verteilt: 69\% arbeiteten Vollzeit, 31\% Teilzeit, mit durchschnittlich 45,2 bzw. 25,7 Arbeitsstunden pro Woche (Tabelle 11). Unter den Teilzeitbeschäftigten gab die Hälfte an, 20 bis 30 Stunden beschäftigt zu sein, relativ viele waren 30 bis 36 Stunden beschäftigt. Unter den teilzeitbeschäftigten Wissenschaftlerinnen waren also 8 von 10 insgesamt 20 oder mehr Stunden beschäftigt, was sich auch in den relativ hohen durchschnittlichen Arbeitsstunden von knapp 26 Wochenstunden widerspiegelt.

Was den Beschäftigungsort betrifft, zeigte sich, dass über ein Viertel teils zu Hause oder ganz zu Hause arbeitete, bei in Teilzeit beschäftigten Frauen arbeiteten verhältnismäßig mehr Frauen teilweise oder ganz von zu Hause aus (31\%) als unter den in Vollzeit Beschäftigten (26\%) (Tabelle A4).

Tabelle 11: Beschäftigungsausmaß und Arbeitsstunden

\begin{tabular}{|l|l|l|l|}
\hline & Abs. & Rel. & Durchschnittliche Arbeitsstunden \\
\hline Vollzeit & 152 & $69 \%$ & 45,2 \\
\hline Teilzeit & 67 & $31 \%$ & 25,7 \\
\hline Total & 219 & $100 \%$ & 39,2 \\
\hline
\end{tabular}

Entsprechend den Angaben zum Beschäftigungsstatus wird es aufgrund der Fallzahlen sinnvoll sein, nur in „Arbeitnehmerinnen“, „Selbständige“" und „Andere“ zu unterscheiden. Nur wenige geringfügig Beschäftigte und Werkvertragsnehmerinnen sind im Wissenschaftlerinnen-Sample enthalten (insgesamt 4\%) (Tabelle 12).

Tabelle 12: Beschäftigungsstatus

\begin{tabular}{|l|l|l|}
\hline & Abs. & Rel. \\
\hline Bezahlte Arbeitnehmerin & 188 & $86 \%$ \\
\hline Geringfügig Beschäftigte & 3 & $1 \%$ \\
\hline Werkvertragsnehmerin, freie Dienstnehmerin & 7 & $3 \%$ \\
\hline Selbständig & 20 & $9 \%$ \\
\hline in einer berufsbezogenen Ausbildung oder Lehre & 1 & $0 \%$ \\
\hline Total & 219 & $100 \%$ \\
\hline
\end{tabular}

Relevant für Analysen könnte auch die Information zur Dauer im aktuellen Job: Zu beachten ist aber wieder die Selektivität des Samples. Ein Viertel der befragten Frauen war fünf Jahre oder länger im aktuellen Job tätig. 
Abbildung 4: Dauer im aktuellen Job

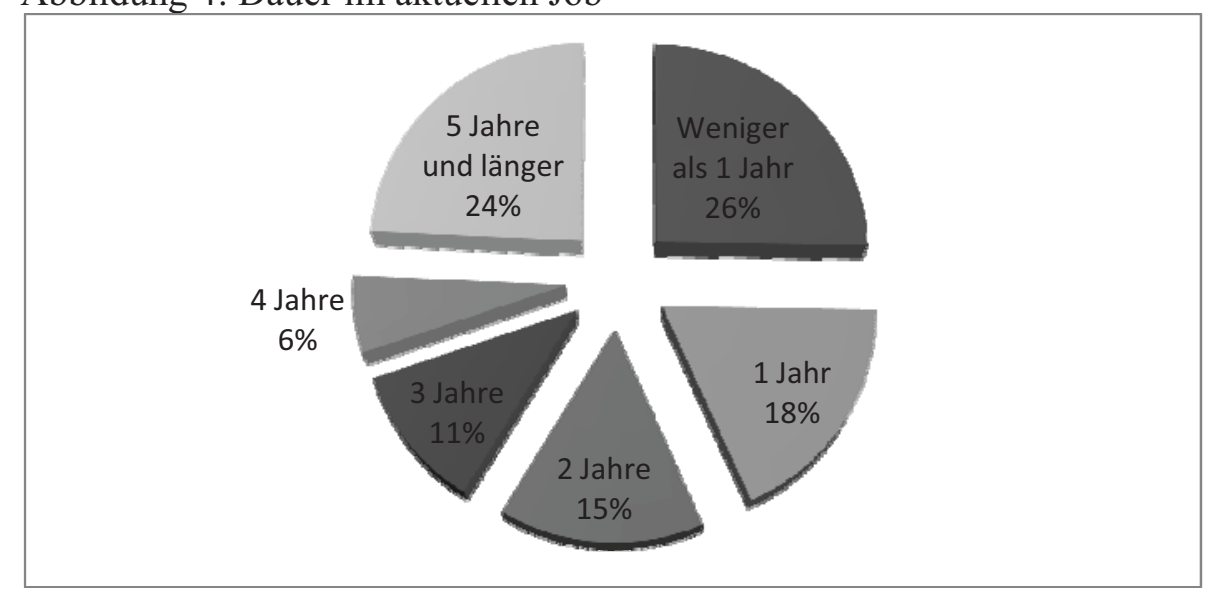

\subsubsection{Zusätzlich erhobene Informationen zur beruflichen Situation}

Bei der Befragung der Wissenschaftlerinnen wurden einige zusätzliche Fragen zur beruflichen Situation aufgenommen, die es ermöglichen sollen, wissenschaftlich tätige Frauen zu identifizieren und ihren Tätigkeitsbereich ${ }^{8}$ näher spezifizieren und analysieren zu können. Aus diesem Grund ist der aktuelle Tätigkeitsbereich der befragten Frauen bekannt (Tabelle 13).

Tabelle 13: Aktueller Tätigkeitsbereich der beschäftigten Frauen

\begin{tabular}{|l|l|l|}
\hline & Abs. & Rel. \\
\hline Hochschulbereich, z.B. Universität oder Fachhochschule & 139 & 63 \\
\hline Außeruniversitäre Forschungseinrichtung & 28 & 13 \\
\hline Forschung und Entwicklung (F\&E) in einem privaten Institut & 5 & 2 \\
\hline Privatwirtschaft (andere als F\&E Tätigkeit) & 19 & 9 \\
\hline Öffentliche Verwaltung & 4 & 2 \\
\hline Schule, Jugend- und Erwachsenenbildung & 5 & 2 \\
\hline Medien, Presse & 3 & 1 \\
\hline Non-Profit Organisationen & 6 & 3 \\
\hline Sonstiges & 10 & 5 \\
\hline Total & 219 & 100 \\
\hline
\end{tabular}

Bemerkung: Im Falle von Mehrfachnennungen wurde die Erstnennung betrachtet.

Die detaillierten Angaben lassen sich zu vier Gruppen zusammenfassen: (1) Hochschulbereich, (2) Forschung, (3) Privatwirtschaft und (4) Anderes. Zur „Forschung“ zählen Tätigkeiten in außeruniversitären Forschungseinrichtungen sowie Forschung und Entwicklung in privaten Instituten. Frauen, die zum Zeitpunkt der Befragung nicht erwerbstätig waren, erhielten analoge Fragen zu ihrer letzten Beschäftigung.

\footnotetext{
${ }^{8}$ Bei der Frage nach dem aktuellen Tätigkeitsbereich waren Mehrfachnennungen möglich für jene Frauen, die mehrere Beschäftigungen angaben. So haben 22 Frauen bei dieser Frage zwei Nennungen codiert, eine Frau hat sogar insgesamt sechs Nennungen.
} 
Abbildung 5: Tätigkeitsbereich der befragten Wissenschaftlerinnen

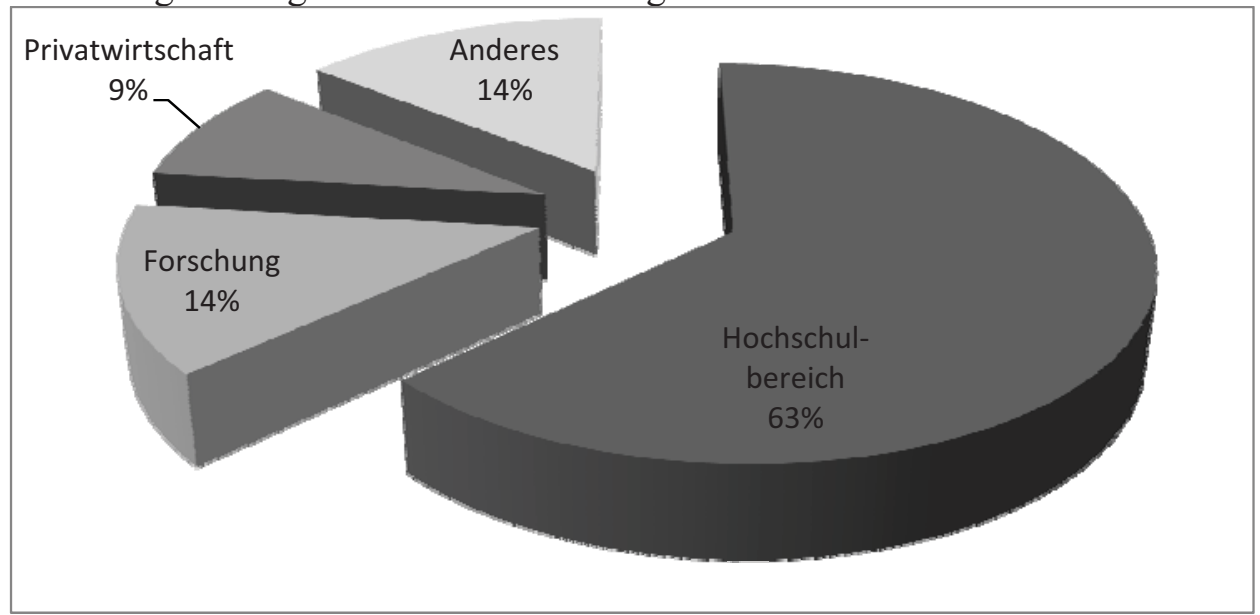

Bemerkung: Aktueller bzw. früherer Tätigkeitsbereich; N=247 Frauen.

Mit knapp zwei Dritteln war der überwiegende Anteil der befragten Wissenschaftlerinnen haupt- oder nebenberuflich im Hochschulbereich tätig. $14 \%$ forschten an einer außeruniversitären Einrichtung oder einem privaten Institut. 9\% waren in der Privatwirtschaft beschäftigt, wobei sie nicht im Bereich Forschung und Entwicklung tätig waren, die verbleibenden 9\% mussten sonstigen Bereichen zugeordnet werden.

\subsubsection{Im Hochschulbereich tätige Frauen}

Insgesamt 156 Frauen waren aktuell oder in ihrer letzten Beschäftigung haupt- oder nebenberuflich im Hochschulbereich tätig. Sie wurden nach der beruflichen Stellung im Hochschulbereich gefragt. Es zeigte sich, dass unter den befragten Frauen 12 ordentliche oder außerordentliche Professorinnen und 9 Dozentinnen waren, weiters wurden 34 Universitätsassistentinnen befragt. Die Hälfte der im Hochschulbereich tätigen Frauen war als wissenschaftliche Mitarbeiterinnen angestellt (Abbildung 6).

Abbildung 6: Berufliche Stellung der im Hochschulbereich tätigen Frauen

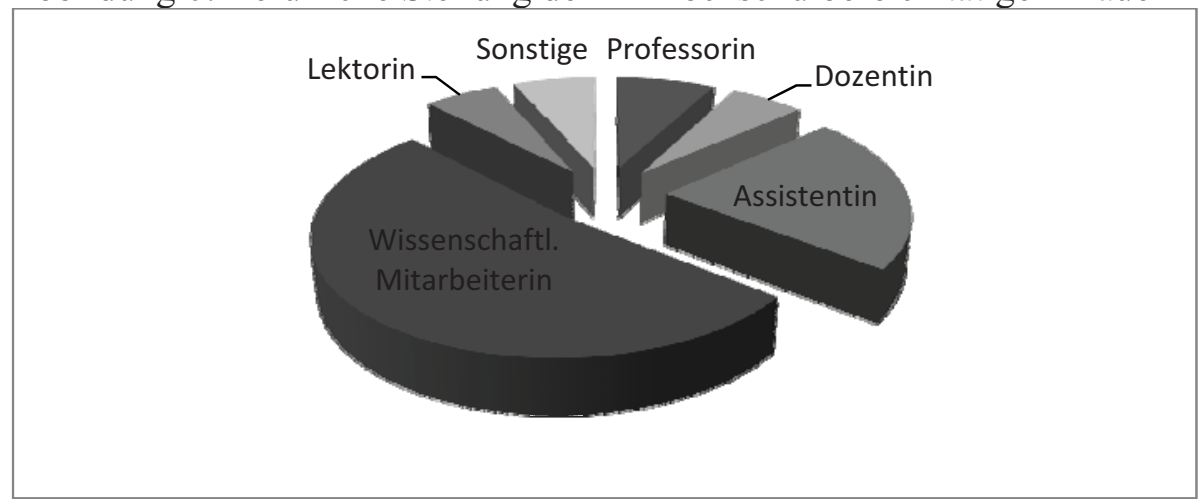




\section{Vergleich der Wissenschaftlerinnen mit den Frauen im Main Sample}

Die Befragung der Wissenschaftlerinnen ist eine Zusatzerhebung im Rahmen des „Generations and Gender Surveys (GGS) 2008/09“, für den österreichweit zwischen September 2008 und Februar 2009 insgesamt 5.000 Männer und Frauen zwischen 18 und 45 Jahren befragt wurden (Buber und Neuwirth, 2009). Diese Hauptbefragung wird in weiterer Folge als Main Sample bezeichnet.

Bei der Analyse des Wissenschaftlerinnen-Samples werden immer wieder Vergleiche mit dem GGS Main Sample von Bedeutung sein. Als mögliche Vergleichsgruppen aus dem Main Sample können folgende zwei Gruppen herangezogen werden: einerseits Akademikerinnen (mit einem ähnliche hohen Bildungsabschluss wie die Wissenschaftlerinnen) und andererseits Nicht-Akademikerinnen, also Frauen mit einem niedrigeren Bildungsabschluss.

Die befragten Wissenschaftlerinnen waren zwischen 25 und 45 Jahre alt. Um einen Vergleich mit dem GGS-Main-Sample anstellen zu können, muss dieses auf Frauen derselben Altersgruppen beschränkt werden. Insgesamt 2.099 Nicht-Akademikerinnen und 354 Akademikerinnen waren zum Zeitpunkt der Befragung zwischen 25 und 45 Jahre alt. Unter Berücksichtigung der Gewichtung ${ }^{9}$ (Buber, 2009) sind dies 2.076 bzw. 377 Frauen $^{10}$. Sie bilden die beiden Vergleichsgruppen.

\subsection{Alter}

Die vorliegenden Berechnungen und Vergleiche basieren somit auf folgenden drei Gruppen: (1) Nicht-Akademikerinnen, (2) Akademikerinnen und (3) Wissenschaftlerinnen. Nach 5-Jahres-Altersgruppen unterteilt ergibt sich folgende Verteilung:

Tabelle 14: Alter der Frauen, Vergleich mit GGS-Grundgesamtheit, absolut

\begin{tabular}{|l|l|l|l|}
\hline & $\begin{array}{l}\text { Nicht- } \\
\text { Akademikerinnen } \\
\text { (GGS) }\end{array}$ & $\begin{array}{l}\text { Akademikerinnen } \\
\text { (GGS) }\end{array}$ & $\begin{array}{l}\text { Wissenschaftlerinnen } \\
\text { (Zusatzsample) }\end{array}$ \\
\hline $25-29$ & 441 & 80 & 72 \\
\hline $30-34$ & 403 & 111 & 90 \\
\hline $35-39$ & 538 & 71 & 49 \\
\hline $40-45$ & 693 & 116 & 36 \\
\hline Total & 2.076 & 377 & 247 \\
\hline
\end{tabular}

Bemerkung: Nicht-Akademikerinnen und Akademikerinnen im GGS wurden mit den VID-Gewichten kalibriert.

\footnotetext{
${ }^{9}$ Das Main Sample wurde mit den vom VID erstellten Gewichten kalibriert, die bei den Frauen nach Alter, Beschäftigungsstatus, Geburtsland, Lebensform und Parität gewichten (Buber, 2009). Für die Wissenschaftlerinnen können keine Gewichte berechnet werden, da die Grundgesamtheit nicht bekannt ist. ${ }^{10}$ Bedingt durch die Tatsache, dass kinderlose Frauen im GGS unterrepräsentiert waren und dass Akademikerinnen in höherem Grad kinderlos waren, ist die gewichtete Gesamtzahl der Akademikerinnen etwas höher als deren ungewichtete Anzahl.
} 
Das Durchschnittsalter der befragten Wissenschaftlerinnen beträgt 33,1 Jahre. Befragte Akademikerinnen und Nichtakademikerinnen im GGS Main Sample waren vergleichsweise älter (35 bzw. 36,7 Jahre). Es zeigt sich, dass die Gruppe der 40- bis 45jährigen Frauen im Wissenschaftlerinnen-Sample relativ gering besetzt ist, da sie mit 15\% anteilsmäßig nur halb so groß ist wie in den beiden Vergleichsgruppen (31\% bzw. 33\%) sind.

Tabelle 15: Alter der Frauen, Vergleich mit GGS-Grundgesamtheit, relativ

\begin{tabular}{|l|l|l|l|}
\hline & $\begin{array}{l}\text { Nicht- } \\
\text { Akademikerinnen } \\
\text { (GGS) }\end{array}$ & $\begin{array}{l}\text { Akademikerinnen } \\
\text { (GGS) }\end{array}$ & $\begin{array}{l}\text { Wissenschaftlerinnen } \\
\text { (Zusatzsample) }\end{array}$ \\
\hline $25-29$ & $21 \%$ & $21 \%$ & $29 \%$ \\
\hline $30-34$ & $19 \%$ & $29 \%$ & $36 \%$ \\
\hline $35-39$ & $26 \%$ & $19 \%$ & $20 \%$ \\
\hline $40-45$ & $33 \%$ & $31 \%$ & $15 \%$ \\
\hline Total & $100 \%$ & $100 \%$ & $100 \%$ \\
\hline
\end{tabular}

Abbildung 7: Altersverteilung der Frauen zwischen 25 und 45, Vergleich mit GGSGrundgesamtheit, relativ

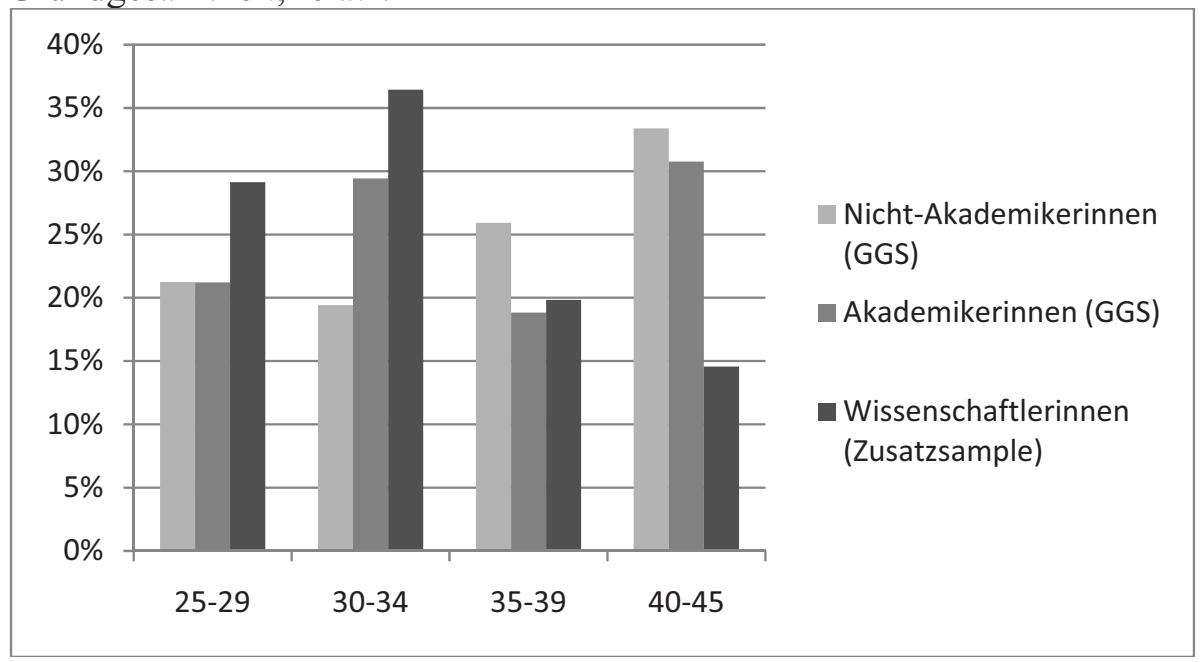

\subsection{Lebensformen}

Der Familienstand, die aktuelle Lebensform und das Vorhandensein eines Partners sind ausschlaggebende Determinanten für die Familiengründung und Familienerweiterung. Der Familienstand, als erster wichtiger Indikator, zeigt einen starken bildungsspezifischen Gradienten: Je höher die Bildungsgruppe, desto niedriger der Anteil der verheirateten Frauen. So waren 53\% der Nicht-Akademikerinnen, 44\% der Akademikerinnen und 29\% der Wissenschaftlerinnen zum Zeitpunkt der Befragung verheiratet (Tabelle 5).

Der Familienstand verliert für die Familiengründung zunehmend an Bedeutung. So ist der Anteil der unehelichen Erstgeburten in Österreich hoch und auch in den letzten Jahren 
weiter gestiegen. Im Jahr 2008 waren 38,8\% aller Geburten und die Hälfte aller Erstgeburten unehelich (Statistik Austria 2009).

Da viele Paare unverheiratet zusammenleben, sind das Vorhandensein eines Partners und die aktuelle Lebensform Indikatoren, die in gewisser Weise die Lebenssituation der Frauen besser zu beschreiben vermögen. Betrachtet man den Anteil der Frauen, die mit einem Partner in einem Haushalt zusammenlebten, so sind die Unterschiede zwischen den drei Vergleichsgruppen wesentlich geringer, als beim Familienstand. Während $70 \%$ der befragten Nicht-Akademikerinnen zwischen 25 und 45 Jahren mit einem Partner zusammenlebten, traf dies weniger oft auf Akademikerinnen (64\%) und Wissenschaftlerinnen zu (62\%) (Abbildung 8). Es gilt: je höher die Bildung, desto niedriger der Anteil der Frauen, die mit einem Partner zusammenleben.

Die oben beschriebenen markanten Unterschiede im Familienstand spiegeln sich vor allem in der formalen Form der Partnerschaft wider: Von den Frauen, die mit ihrem Partner zusammen-wohnten, lebten vergleichsweise wenige Nicht-Akademikerinnen $(25 \%)$ und mehr Akademikerinnen (34\%) und Wissenschaftlerinnen (56\%) in einer nichteheliche Lebensgemeinschaft. Zusammenfassend kann also gesagt werden, dass NichtAkademikerinnen häufiger mit einem Partner zusammenleben als Akademikerinnen und Wissenschaftlerinnen, und dass sie viel öfter mit ihrem Partner verheiratet sind. Unter höher gebildeten Frauen ist die nichteheliche Partnerschaft eine üblichere Partnerschaftsform, besonders unter den befragten Wissenschaftlerinnen. Bei wem das nichteheliche Zusammenleben in eine Ehe übergeht, kann nicht gesagt werden.

Tabelle 16: Familienstand, Vergleich mit GGS-Grundgesamtheit

\begin{tabular}{|l|l|l|l|}
\hline & $\begin{array}{l}\text { Nicht-Akademikerinnen } \\
(\mathrm{GGS})\end{array}$ & $\begin{array}{l}\text { Akademikerinnen } \\
(\mathrm{GGS})\end{array}$ & $\begin{array}{l}\text { Wissenschaftlerinnen } \\
\text { (Zusatzsample) }\end{array}$ \\
\hline Verheiratet & $53 \%$ & $44 \%$ & $29 \%$ \\
\hline Geschieden & $11 \%$ & $4 \%$ & $1 \%$ \\
\hline Verwitwet & $1 \%$ & $0 \%$ & $0 \%$ \\
\hline Ledig & $36 \%$ & $52 \%$ & $70 \%$ \\
\hline Total & $100 \%$ & $100 \%$ & $100 \%$ \\
\hline
\end{tabular}

Der Anteil der befragten Wissenschaftlerinnen, die zwar einen Partner hat, mit diesem aber nicht zusammenlebt, ist relativ hoch (21\%) (Abbildung 8). Bei den Gründen für eine sogenannte Living Apart Together-Beziehung (LAT-Beziehung) wurden von Wissenschaftlerinnen und Akademikerinnen vorrangig äußere Umstände, und vor allem berufliche Belange angegeben. Nicht-Akademikerinnen nannten für das Getrenntleben vorrangig wohnungsbedingte Gründe. Wissenschaftlerinnen unterscheiden sich von Akademikerinnen und Nicht-Akademikerinnen insofern, als dass ein höherer Anteil angibt, dass beide Partner getrennt leben möchten (31\% versus $23 \%$ und $17 \%$ ). 
Abbildung 8: Partnerschaft, Vergleich mit GGS-Grundgesamtheit, relativ

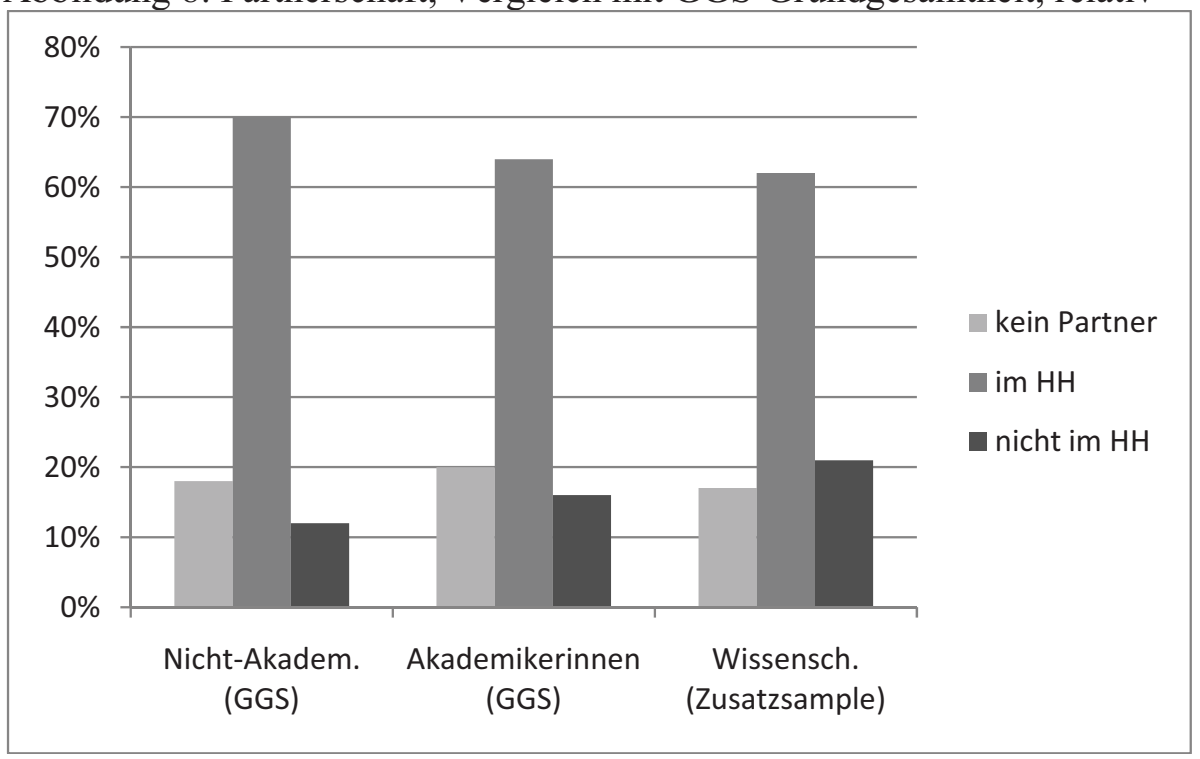

\subsection{Geborene Kinder}

Familiengründung und gewünschte sowie realisierte Kinderzahl sind zentrale Aspekte der GGS-Befragung. In der vorliegenden Arbeit wird kurz eine erste Übersicht über die Anzahl der geborenen Kinder gegeben.

Es zeigt sich, dass die befragten Wissenschaftlerinnen sehr wenige Kinder haben. Die durchschnittliche Kinderzahl ist nur ein Drittel so hoch wie die der NichtAkademikerinnen. So haben die befragten Wissenschaftlerinnen durchschnittlich 0,47 Kinder, während die Nicht-Akademikerinnen der gleichen Altersgruppe durchschnittlich 1,39 Kinder geboren haben. Auch liegt die Kinderzahl deutlich unter jener der Akademikerinnen $(0,76)$ des GGS-Samples.

Abbildung 9: Durchschnittliche Anzahl der leiblichen Kinder

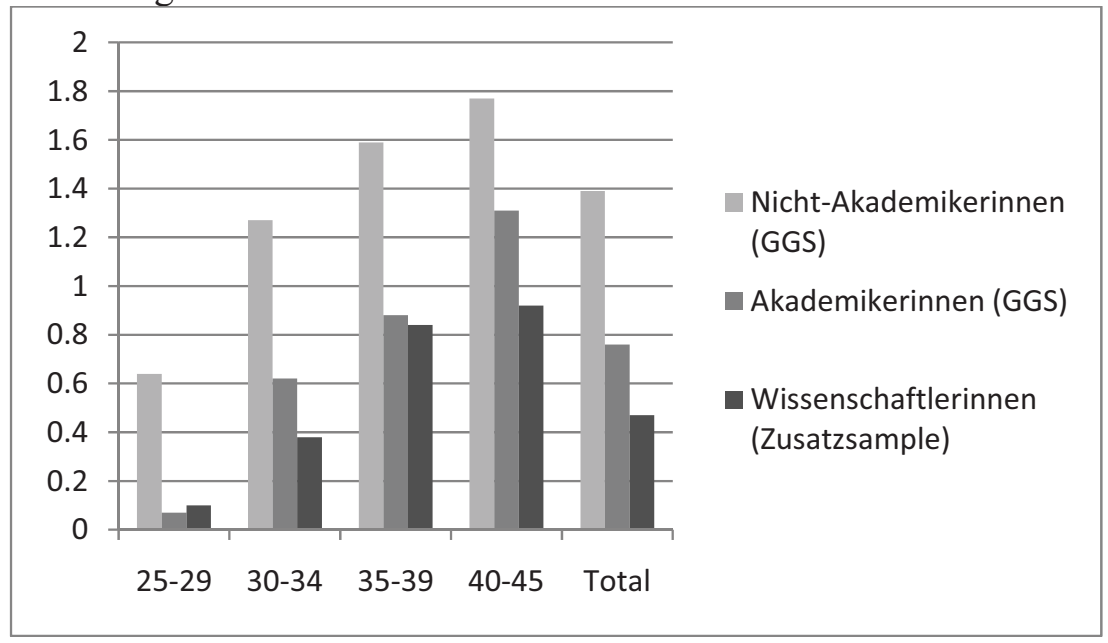


Unterschieden nach Paritäten ergibt sich folgendes Bild: Kinderlosigkeit war unter den befragten Wissenschaftlerinnen mit 69\% sehr hoch. Im Vergleich dazu waren 55\% der Akademikerinnen aber nur 29\% der Nicht-Akademikerinnen zum Zeitpunkt der Befragung kinderlos.

$18 \%$ der Wissenschaftlerinnen hatten ein Kind, 11\% zwei Kinder, nur wenige (2\%) hatten 3 Kinder, eine einzige Frau vier Kinder. Bei den Akademikerinnen hatten 5\% drei oder mehr Kinder, bei den Nicht-Akademikerinnen 15\%. Die befragten Wissenschaftlerinnen waren somit in hohem Maße kinderlos. Wenige hatten eine Familie mit zumeist einem oder zwei Kindern (Tabelle A7).

Erwartungsgemäß waren Frauen mit höherem Bildungsgrad bei der Geburt ihres ersten Kindes durchschnittlich älter. So bekamen Nicht-Akademikerinnen im Durchschnitt mit 24,8 Jahren ihr erstes Kind, Akademikerinnen mit 29,3 und die befragten Wissenschaftlerinnen mit 30,2 Jahren.

\subsection{Kinderwunsch}

Wie viele Kinder wünschen sich die befragten Wissenschaftlerinnen? Die Beantwortung dieser Frage unter Berücksichtigung verschiedener persönlicher wie berufsspezifischer Faktoren ist ein Ziel der laufenden Arbeiten am Vienna Institute of Demography (VID). Erste Ergebnisse zum Kinderwunsch ${ }^{11}$ werden hier nur deskriptiv angeführt.

Geht man von der angegebenen Zahl aus, so ist der Kinderwunsch der befragten Wissenschaftlerinnen niedriger als bei Nicht-Akademikerinnen, die gewünschten Kinderzahlen nach Kohorten sind aber - in Bezug auf die Zahl der bislang geborenen Kinder - relativ hoch. Der Kinderwunsch der Akademikerinnen im GGS Main Sample und der befragten Wissenschaftlerinnen verhält sich ähnlich und liegt unter dem von niedriger gebildeten Frauen (Abbildung 10).

\footnotetext{
${ }^{11}$ Es ist zu beachten, dass im GGS an Personen mit gleichgeschlechtlicher Partnerin bzw. gleichgeschlechtlichem Partner nicht Fragen zur Fertilität gestellt wurden. Damit können jene 10 befragten Wissenschaftlerinnen, die angaben, eine gleichgeschlechtliche Partnerin zu haben, nicht zur Analyse des Kinderwunsches herangezogen werden. Einige Frauen gaben keine Angaben zum Kinderwunsch. Es verbleiben 234 Wissenschaftlerinnen mit detaillierten Angaben zum Kinderwunsch.
} 
Abbildung 10: Vergleich des Kinderwunsches bei Nicht-Akademikerinnen, Akademikerinnen und Wissenschaftlerinnen

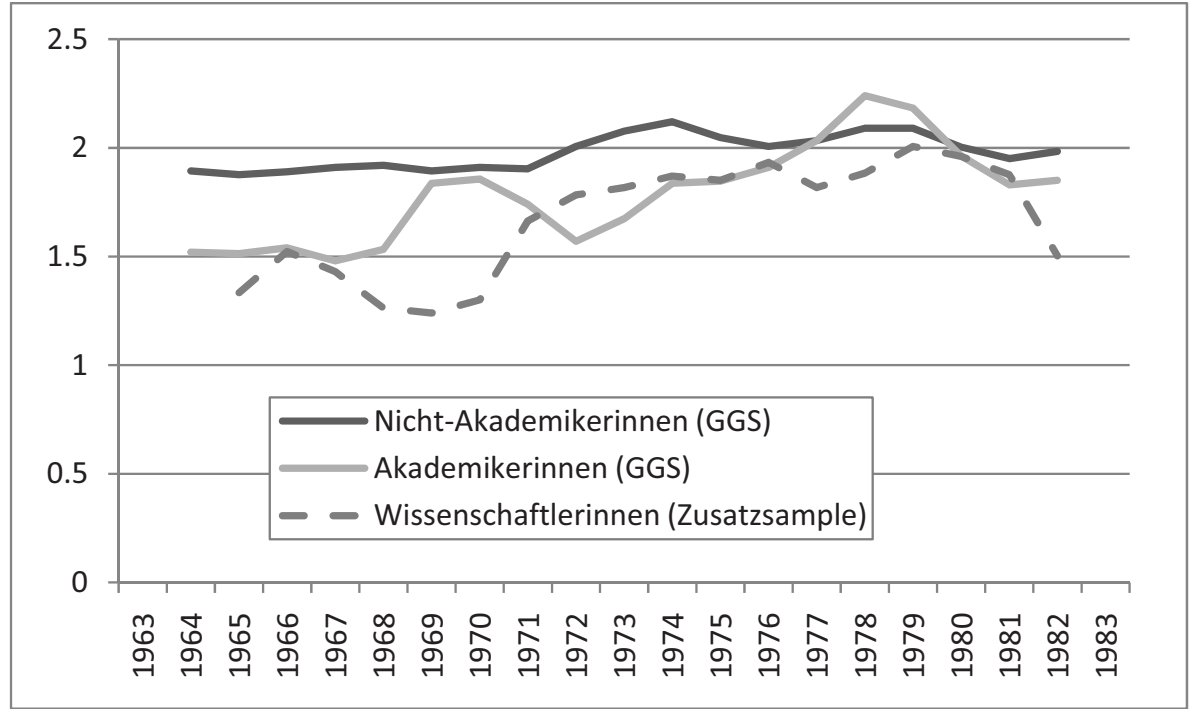

Bemerkung 1: Geglättete Durchschnitte von jeweils drei Kohorten

Bemerkung 2: Aufgrund der geringen Fallzahlen (Tabelle A8) ergeben sich bei den Akademikerinnen und Wissenschaftlerinnen relativ große Schwankungen.

\subsection{Geborene und zusätzlich gewünschte Kinderzahl}

Wissenschaftlerinnen ab 35 Jahren wünschen sich deutlich weniger Kinder als gleichaltrige Nicht-Akademikerinnen. Ein Vergleich der bereits geborenen und noch zusätzlich gewünschten Kinder nach Altersgruppen der Frauen bringt ein interessantes Phänomen zutage: Während die Unterschiede bei den bereits geborenen Kindern groß sind, sind die Unterschiede bei der insgesamt gewünschten Kinderzahl deutlich weniger stark ausgeprägt (Abbildung 11).

Mit zunehmendem Alter unterscheiden sich die Akademikerinnen aus dem GGS-Sample von den befragten Wissenschaftlerinnen nicht nur in der Anzahl der geborenen Kinder, sondern auch in der gewünschten Kinderzahl. Auffallend groß ist der Unterschied zwischen befragten Wissenschaftlerinnen und Akademikerinnen Anfang 40: Während Akademikerinnen zwischen 40 und 45 Jahren im Durchschnitt 1,33 Kinder geboren haben und mit den noch zusätzlich gewünschten Kindern ein Niveau von 1,52 anstreben, haben die befragten Wissenschaftlerinnen zwischen 40 und 45 Jahren im Schnitt 0,97 Kinder geboren. Werden die noch gewünschten Kinder hinzugerechnet, so kommen sie auf 1,35 als gewünschte Kinderzahl.

In der höchsten Altersgruppe der befragten Frauen, also unter Frauen zwischen 40 und 45 Jahren, die am Ende ihrer reproduktiven Phase sind, werden Unterschiede von NichtAkademikerinnen zu höher gebildeten Frauen sehr markant. Nicht-Akademikerinnen zwischen 40 und 45 Jahren haben 1,77 Kinder geboren, mit den noch zusätzlich gewünschten Kindern kämen sie auf 1,91 Kinder. 
Abbildung 11: Geborene Kinder und zusätzlicher Kinderwunsch, Vergleich von NichtAkademikerinnen, Akademikerinnen und Wissenschaftlerinnen

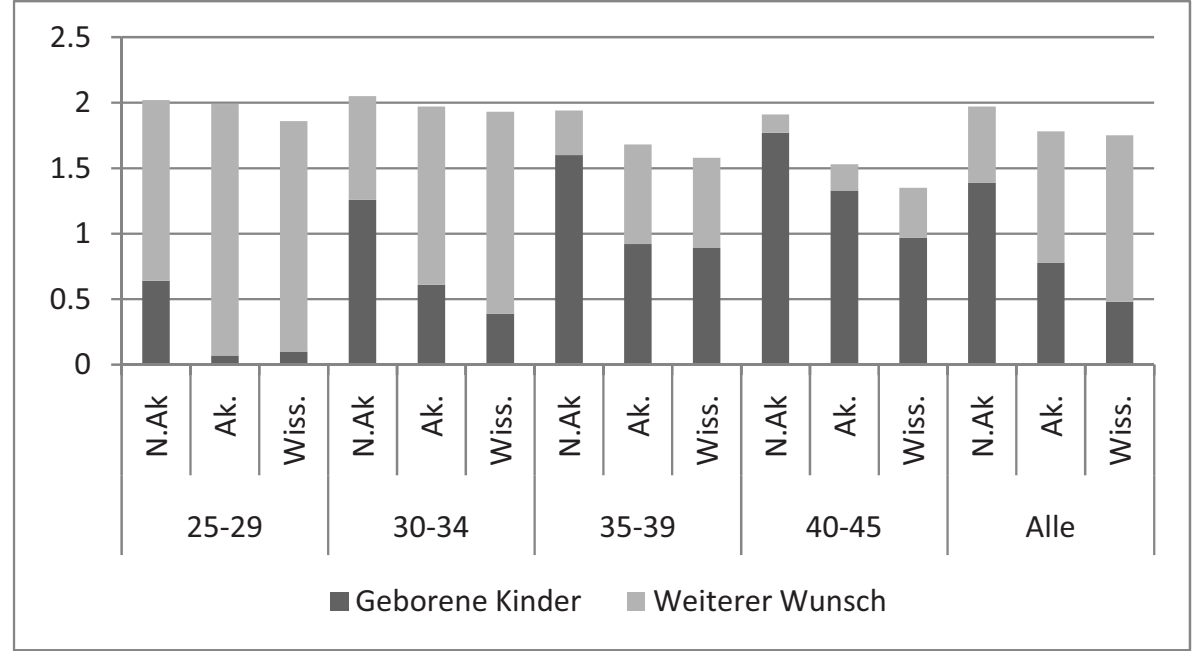

\subsection{Kinderlose Frauen}

Werden nur die Kinderlosen betrachtet, so zeigt sich, dass acht von zehn befragten kinderlosen Wissenschaftlerinnen und Akademikerinnen - wahrscheinlich oder ganz sicher - ein Kind haben möchten. Relativ viele der befragten kinderlosen Wissenschaftlerinnen denken an eine späte Mutterschaft: So möchte die Hälfte aller kinderlosen Wissenschaftlerinnen zwischen 40 und 45 ein Kind (Tabelle 17). Unter Akademikerinnen und Nicht-Akademikerinnen ist der späte Kinderwunsch weniger stark ausgeprägt $(35 \%$ bzw. 27\%). Allgemein ist der Wunsch nach Kindern relativ stark ausgeprägt, denn der Großteil der kinderlosen Frauen über 40, die sich ein Kind wünschen, möchte dies ,ganz sicher". Eine mögliche Interpretation ist, dass höher gebildete Frauen eine späte Mutterschaft eher in Betracht ziehen als niedriger gebildete. Möglicherweise ziehen sie auch eher medizinische Hilfe in Betracht, um auch noch nach 40 eine Familie gründen zu können. Inwiefern sie ihren Kinderwunsch realisieren können ist fraglich, da mit zunehmendem Alter die Fekundität von Frauen stark abnimmt.

Tabelle 17: Kinderwunsch der kinderlosen Frauen, Anteil der Frauen die wahrscheinlich oder ganz sicher ein Kind möchten

\begin{tabular}{|l|l|l|l|}
\hline & N-Ak. & Ak. & Wiss. \\
\hline $25-29$ & $89 \%$ & $91 \%$ & $89 \%$ \\
\hline $30-34$ & $83 \%$ & $88 \%$ & $90 \%$ \\
\hline $35-39$ & $55 \%$ & $87 \%$ & $55 \%$ \\
\hline $40-45$ & $27 \%$ & $35 \%$ & $50 \%$ \\
\hline Total & $71 \%$ & $80 \%$ & $82 \%$ \\
\hline
\end{tabular}




\section{Ausblick}

Derzeit wird am VID intensiv mit den Daten des sogenannten WissenschaftlerinnenSamples gearbeitet. Ergebnisse multivariater Analysen sind für Ende 2010 geplant. 


\section{Literatur}

Armenti, Carmen (2004) Women faculty seeking tenure and parenthood: lessons from previous generations, Cambridge Journal of Education 34(1), 65-83.

Buber, Isabella (2009) Parity-specific weights for the Austrian Generations and Gender Survey. Reference Paper. Vienna Institute of Demography.

Buber, Isabella und Norbert Neuwirth (Hg.) (2009) Familienentwicklung in Österreich. Erste Ergebnisse des "Generations and Gender Survey (GGS)" 2008/09, Wien. URL: http://www.ggp-austria.at/familienentwicklung.pdf

Eurostat (1999) Handbuch der Bildungs- und Ausbildungsfelder, URL: http://www.statistik.at/web de/klassifikationen/klassifikationsdatenbank/weitere klassifika tionen/bildungsklassifikation/index.html, 4 Mai 2010.

Fox, Mary Frank (2005) Gender, family characteristics and publication productivity among scientists, Social Studies of Science 35, 131-150.

Kemkes-Grottenthaler, Ariane (2003) Postponing or rejecting parenthood? Results of a survey among female academic professionals, Journal of Biosocial Science 35, 213-226.

Lampic, Claudia, Agneta Skoog Svanberg, Per-Olov Karlström und Tanja Tydén (2005) Fertility awareness, intentions concerning childbearing and attitudes towards parenthood among female and male academics, Human Reproduction 21(2), 558-564.

Lind, Inken (2008) Dossier Balancierung von Wissenschaft und Elternschaft, Auswahl erster Ergebnisse der Online-Befragung, URL: http://www.bawie.de/web/files/49/de/Ergebnisbericht.pdf, 29 April 2010.

Romanin, Susanne und Ray Over (1993) Australien academics: career patterns, work roles, and family life-cycle commitments of men and women, Higher Education 26(4), 411-429.

ÖAW (2008) Köpfe, Stipendien und Preise. Bericht 2008, Wien.

Statistik Austria (2009) Demographisches Jahrbuch 2008, Wien.

Van Anders, Sari M. (2004) Why the academic pipeline leaks: fewer men than women perceive barriers to becoming professors, Sex Roles 51(9/10), 511-521.

Wolf-Wende, Lisa Ellen und Kelly Ward (2006) Academic life and motherhood: variations by institutional type, Higher Education 52, 487-521. 


\section{Tabellen}

\subsection{Tabellen zu den Abbildungen}

Tabelle zu Abbildung 1: Umfragebereitschaft unter den nachweislich kontaktierten Frauen, nach Status des Stipendienantrags

\begin{tabular}{|l|l|l|l|l|l|}
\hline Status & Zusage & Absage & keine Antwort & Total & N \\
\hline Altstipendiatin & $38 \%$ & $1 \%$ & $62 \%$ & $100 \%$ & 221 \\
\hline Antragstellerin & $18 \%$ & $0 \%$ & $82 \%$ & $100 \%$ & 828 \\
\hline Stipendiatin & $51 \%$ & $0 \%$ & $49 \%$ & $100 \%$ & 90 \\
\hline Total & $25 \%$ & $0 \%$ & $75 \%$ & $100 \%$ & 1139 \\
\hline
\end{tabular}

Tabelle zur Abbildung 2: Altersverteilung in der Grundgesamtheit, unter den nachweislich kontaktierten und den tatsächlich befragten Frauen

\begin{tabular}{|l|l|l|l|l|l|l|}
\hline & \multicolumn{2}{|l|}{ absolut } & relativ \\
\hline & Grundgesamtheit & Kontaktiert & Interviewt & Grundgesamtheit & Kontaktiert & Interviewt \\
\hline $1964-1969$ & 236 & 163 & 38 & $15 \%$ & $14 \%$ & $15 \%$ \\
\hline $1970-1974$ & 430 & 287 & 54 & $28 \%$ & $25 \%$ & $22 \%$ \\
\hline $1975-1979$ & 623 & 450 & 94 & $40 \%$ & $40 \%$ & $38 \%$ \\
\hline $1980-1984$ & 272 & 239 & 61 & $17 \%$ & $21 \%$ & $25 \%$ \\
\hline Total & 1561 & 1139 & 247 & $100 \%$ & $100 \%$ & $100 \%$ \\
\hline
\end{tabular}

Grundgesamtheit: 1,561 Adressen; Kontaktiert: 1139 Frauen; Befragt: 247 Frauen

Tabelle zu Abbildung 3: Anteil der Stipendiatinnen und Altstipendiatinnen in der Grundgesamtheit, unter den kontaktierten Frauen und den interviewten Frauen, nach Stipendienart

\begin{tabular}{|l|l|l|l|}
\hline & Grundgesamtheit & Kontaktiert & Interviewt \\
\hline Apart & $27 \%$ & $31 \%$ & $39 \%$ \\
\hline DOC & $22 \%$ & $23 \%$ & $44 \%$ \\
\hline DOC-TEAM & $55 \%$ & $58 \%$ & $71 \%$ \\
\hline DOC fFORTE & $31 \%$ & $31 \%$ & $50 \%$ \\
\hline Rom & $42 \%$ & $40 \%$ & $50 \%$ \\
\hline Total & $26 \%$ & $27 \%$ & $47 \%$ \\
\hline
\end{tabular}

Tabelle zu Abbildung 4: Dauer im aktuellen Job

\begin{tabular}{|l|l|}
\hline Dauer & Rel. \\
\hline Weniger als 1 Jahr & $25 \%$ \\
\hline 1 Jahr & $18 \%$ \\
\hline 2 Jahre & $15 \%$ \\
\hline 3 Jahre & $11 \%$ \\
\hline 4 Jahre & $6 \%$ \\
\hline 5 Jahre und länger & $24 \%$ \\
\hline Total & $100 \%$ \\
\hline
\end{tabular}


Tabelle zu Abbildung 5: Tätigkeitsbereich der befragten Wissenschaftlerinnen

\begin{tabular}{|l|l|l|}
\hline & Abs. & Rel. \\
\hline Hochschulbereich & 156 & 63 \\
\hline Forschung & 34 & 14 \\
\hline Privatwirtschaft & 22 & 9 \\
\hline Anderes & 35 & 14 \\
\hline Total & 247 & 100 \\
\hline
\end{tabular}

Tabelle zu Abbildung 6: Berufliche Stellung der im Hochschulbereich tätigen Frauen

\begin{tabular}{|l|l|}
\hline & Abs. \\
\hline Professorin & 8 \\
\hline a.o. Professorin & 4 \\
\hline Dozentin / Privatdozentin & 9 \\
\hline Assistentin & 34 \\
\hline Wissenschaftliche Mitarbeiterin & 81 \\
\hline Lektorin & 9 \\
\hline Sonstiges & 10 \\
\hline Total & 156 \\
\hline
\end{tabular}

Tabelle zu Abbildung 7: Berufliche Stellung der im Hochschulbereich tätigen Frauen

\begin{tabular}{|l|l|l|l|}
\hline & $\begin{array}{l}\text { Nicht-Akademikerinnen } \\
\text { (GGS) }\end{array}$ & $\begin{array}{l}\text { Akademikerinnen } \\
\text { (GGS) }\end{array}$ & $\begin{array}{l}\text { Wissenschaftlerinnen } \\
\text { (Zusatzsample) }\end{array}$ \\
\hline $25-29$ & $21 \%$ & $21 \%$ & $29 \%$ \\
\hline $30-34$ & $19 \%$ & $29 \%$ & $36 \%$ \\
\hline $35-39$ & $26 \%$ & $19 \%$ & $20 \%$ \\
\hline $40-45$ & $33 \%$ & $31 \%$ & $15 \%$ \\
\hline
\end{tabular}

Tabelle zu Abbildung 8: Partnerschaft, Vergleich mit GGS-Grundgesamtheit

\begin{tabular}{|l|l|l|l|}
\hline & $\begin{array}{l}\text { Nicht-Akademikerinnen } \\
\text { (GGS) }\end{array}$ & $\begin{array}{l}\text { Akademikerinnen } \\
\text { (GGS) }\end{array}$ & $\begin{array}{l}\text { Wissenschaftlerinnen } \\
\text { (Zusatzsample) }\end{array}$ \\
\hline kein Partner & $18 \%$ & $20 \%$ & $17 \%$ \\
\hline im HH & $70 \%$ & $64 \%$ & $62 \%$ \\
\hline nicht im HH & $12 \%$ & $16 \%$ & $21 \%$ \\
\hline Total & $100 \%$ & $100 \%$ & $100 \%$ \\
\hline
\end{tabular}

Tabelle zu Abbildung 9: Durchschnittliche Anzahl der leiblichen Kinder

\begin{tabular}{|l|l|l|l|}
\hline & $\begin{array}{l}\text { Nicht-Akademikerinnen } \\
\text { (GGS) }\end{array}$ & $\begin{array}{l}\text { Akademikerinnen } \\
\text { (GGS) }\end{array}$ & $\begin{array}{l}\text { Wissenschaftlerinnen } \\
\text { (Zusatzsample) }\end{array}$ \\
\hline $25-29$ & 0,64 & 0,07 & 0,1 \\
\hline $30-34$ & 1,27 & 0,62 & 0,38 \\
\hline $35-39$ & 1,59 & 0,88 & 0,84 \\
\hline $40-45$ & 1,77 & 1,31 & 0,92 \\
\hline Total & 1,39 & 0,76 & 0,47 \\
\hline
\end{tabular}


Tabelle zu Abbildung 10: Kinderwunsch, mittlere Variante, nach Bildungsgruppen

\begin{tabular}{|l|l|l|l|}
\hline & $\begin{array}{l}\text { Nicht-Akademikerinnen } \\
\text { (GGS) }\end{array}$ & $\begin{array}{l}\text { Akademikerinnen } \\
\text { (GGS) }\end{array}$ & $\begin{array}{l}\text { Wissenschaftlerinnen } \\
\text { (Zusattzssample) }\end{array}$ \\
\hline 1964 & 1,89 & 1,52 & \\
\hline 1965 & 1,88 & 1,51 & 1,33 \\
\hline 1966 & 1,89 & 1,54 & 1,52 \\
\hline 1967 & 1,91 & 1,48 & 1,43 \\
\hline 1968 & 1,92 & 1,53 & 1,26 \\
\hline 1969 & 1,89 & 1,84 & 1,24 \\
\hline 1970 & 1,91 & 1,86 & 1,30 \\
\hline 1971 & 1,90 & 1,74 & 1,66 \\
\hline 1972 & 2,01 & 1,57 & 1,78 \\
\hline 1973 & 2,08 & 1,67 & 1,82 \\
\hline 1974 & 2,12 & 1,84 & 1,87 \\
\hline 1975 & 2,05 & 1,85 & 1,85 \\
\hline 1976 & 2,01 & 1,91 & 1,93 \\
\hline 1977 & 2,03 & 2,03 & 1,82 \\
\hline 1978 & 2,09 & 2,24 & 1,88 \\
\hline 1979 & 2,09 & 2,18 & 2,01 \\
\hline 1980 & 2,00 & 1,96 & 1,96 \\
\hline 1981 & 1,95 & 1,83 & 1,88 \\
\hline 1982 & 1,98 & 1,85 & 1,50 \\
\hline Total & 1,85 & 1,75 & 1,15 \\
\hline
\end{tabular}

Bemerkung 1: Geglättete Durchschnitte von jeweils drei Kohorten

Tabelle zu Abbildung 11: Geborene Kinder und zusätzlicher Kinderwunsch, Vergleich von Nicht-Akademikerinnen, Akademikerinnen und Wissenschaftlerinnen

\begin{tabular}{|l|l|l|l|l|}
\hline & & Geborene Kinder & Weiterer Wunsch & Kinderwunsch insg. \\
\hline $25-29$ & N.Ak & 0,64 & 1,38 & 2,02 \\
\hline & Ak. & 0,07 & 1,92 & 1,99 \\
\hline & Wiss. & 0,1 & 1,76 & 1,86 \\
\hline $30-34$ & N.Ak & 1,26 & 0,79 & 2,05 \\
\hline & Ak. & 0,61 & 1,36 & 1,98 \\
\hline & Wiss. & 0,39 & 1,54 & 1,93 \\
\hline $35-39$ & N.Ak & 1,6 & 0,34 & 1,94 \\
\hline & Ak. & 0,92 & 0,76 & 1,68 \\
\hline & Wiss. & 0,89 & 0,69 & 1,58 \\
\hline $40-45$ & N.Ak & 1,77 & 0,14 & 1,91 \\
\hline & Ak. & 1,33 & 0,2 & 1,52 \\
\hline & Wiss. & 0,97 & 0,38 & 1,35 \\
\hline Alle & N.Ak & 1,39 & 0,58 & 1,97 \\
\hline & Ak. & 0,78 & 1 & 1,78 \\
\hline & Wiss. & 0,48 & 1,27 & 1,76 \\
\hline
\end{tabular}




\subsection{Zusätzliche Tabellen}

Tabelle A1: Antwortbereitschaft nach Art des beantragten Stipendiums

\begin{tabular}{|l|l|l|l|l|l|l|l|}
\hline Stipendienart & Zusage & Absage & Ausland & nicht erreicht & keine Antwort & Total & $\mathrm{N}$ \\
\hline Apart & $25 \%$ & $0 \%$ & $7 \%$ & $18 \%$ & $50 \%$ & $100 \%$ & 199 \\
\hline DOC & $14 \%$ & $0 \%$ & $4 \%$ & $27 \%$ & $55 \%$ & $100 \%$ & 996 \\
\hline DOC-TEAM & $45 \%$ & $0 \%$ & $5 \%$ & $5 \%$ & $45 \%$ & $100 \%$ & 44 \\
\hline DOC fFORTE & $23 \%$ & $0 \%$ & $3 \%$ & $16 \%$ & $57 \%$ & $100 \%$ & 286 \\
\hline Rom & $22 \%$ & $0 \%$ & $3 \%$ & $14 \%$ & $61 \%$ & $100 \%$ & 36 \\
\hline Total & $18 \%$ & $0 \%$ & $4 \%$ & $23 \%$ & $55 \%$ & $100 \%$ & 1561 \\
\hline
\end{tabular}

Tabelle A2: Antwortbereitschaft der nachweislich kontaktierten Frauen nach Art des beantragten Stipendiums

\begin{tabular}{|l|l|l|l|l|l|}
\hline Stipendienart & Zusage & Absage & keine Antwort & Total & N \\
\hline Apart & $33 \%$ & $0 \%$ & $67 \%$ & 100 & 150 \\
\hline DOC & $20 \%$ & $0 \%$ & $80 \%$ & 100 & 690 \\
\hline DOC-TEAM & $50 \%$ & $0 \%$ & $50 \%$ & 100 & 40 \\
\hline DOC fFORTE & $29 \%$ & $0 \%$ & $71 \%$ & 100 & 229 \\
\hline Rom & $27 \%$ & $0 \%$ & $73 \%$ & 100 & 30 \\
\hline Total & $25 \%$ & $0 \%$ & $75 \%$ & 100 & 1139 \\
\hline
\end{tabular}

Tabelle A3: Beginn und Ende der angetretenen Stipendien

\begin{tabular}{|l|l|l|l|l|l|l|l|}
\hline \multicolumn{3}{|l|}{ Anträge } & \multicolumn{3}{l|}{ Stipendienbeginn } & \multicolumn{3}{l|}{ Stipendienende } \\
\hline Beginn & Abs, & Cum, in $\%$ & Abs, & Cum, in $\%$ & Ende & Abs, & Cum, in $\%$ \\
\hline 1993 & 1 & $0 \%$ & 1 & $1 \%$ & 1996 & 1 & $1 \%$ \\
\hline 1995 & 2 & $1 \%$ & 1 & $2 \%$ & 1997 & 1 & $2 \%$ \\
\hline 1996 & 12 & $6 \%$ & 3 & $5 \%$ & 1998 & 5 & $6 \%$ \\
\hline 1997 & 8 & $9 \%$ & 4 & $8 \%$ & 1999 & 1 & $7 \%$ \\
\hline 1998 & 4 & $11 \%$ & 6 & $14 \%$ & 2000 & 4 & $11 \%$ \\
\hline 1999 & 8 & $14 \%$ & 1 & $14 \%$ & 2001 & 2 & $13 \%$ \\
\hline 2000 & 3 & $15 \%$ & 3 & $17 \%$ & 2002 & 2 & $14 \%$ \\
\hline 2001 & 11 & $20 \%$ & 5 & $22 \%$ & 2003 & 5 & $19 \%$ \\
\hline 2002 & 12 & $25 \%$ & 8 & $29 \%$ & 2004 & 11 & $29 \%$ \\
\hline 2003 & 20 & $33 \%$ & 6 & $34 \%$ & 2005 & 8 & $36 \%$ \\
\hline 2004 & 18 & $40 \%$ & 7 & $41 \%$ & 2006 & 9 & $44 \%$ \\
\hline 2005 & 27 & $51 \%$ & 15 & $54 \%$ & 2007 & 9 & $52 \%$ \\
\hline 2006 & 45 & $69 \%$ & 11 & $64 \%$ & 2008 & 15 & $66 \%$ \\
\hline 2007 & 37 & $84 \%$ & 19 & $81 \%$ & 2009 & 11 & $76 \%$ \\
\hline 2008 & 39 & $100 \%$ & 21 & $100 \%$ & 2010 & 20 & $94 \%$ \\
\hline Total & 247 & & 111 & & 2011 & 7 & $100 \%$ \\
\hline & & & & & Total & 111 & \\
\hline
\end{tabular}


Tabelle A4: Arbeitsort der Beschäftigung

\begin{tabular}{|l|l|l|l|l|l|}
\hline & No, & $\%$ & & Vollzeit & Teilzeit \\
\hline Auswärts an einem Ort & 138 & $63 \%$ & & $66 \%$ & $57 \%$ \\
\hline Zu Hause & 14 & $6 \%$ & & $4 \%$ & $12 \%$ \\
\hline Teils zu Hause, teils auswärts & 46 & $21 \%$ & & $22 \%$ & $19 \%$ \\
\hline Auswärts an verschiedenen Orten & 20 & $9 \%$ & & $8 \%$ & $12 \%$ \\
\hline Weiß nicht & 1 & $0 \%$ & & $1 \%$ & $0 \%$ \\
\hline Total & 219 & $100 \%$ & & 152 & 67 \\
\hline
\end{tabular}


Tabelle A5: Berufsbezeichnung der aktuellen Tätigkeit (ISCO-88-Codierung)

\begin{tabular}{|c|c|}
\hline & Abs. \\
\hline Generaldirektoren und Hauptgeschäftsführer & 1 \\
\hline Produktions- und Operationsleiter, anderweitig nicht genannt & 9 \\
\hline Werbeleiter und Leiter der Öffentlichkeitsarbeit & 1 \\
\hline Sonstige Fachbereichsleiter, anderweitig nicht genannt & 1 \\
\hline Leiter kleiner Unternehmen, anderweitig nicht genannt & 2 \\
\hline Chemiker & 2 \\
\hline Mathematiker und verwandte Berufe & 2 \\
\hline Architekten, Diplomingenieure und verwandte Berufe & 7 \\
\hline Architekten, Raum- und Verkehrsplaner & 1 \\
\hline Diplomingenieure Bauwesen & 1 \\
\hline Biologen, Botaniker, Zoologen und verwandte Wissenschaftler & 9 \\
\hline Pharmakologen, Pathologen und verwandte Wissenschaftler & 2 \\
\hline Ärzte & 1 \\
\hline Tierärzte & 1 \\
\hline Universitäts- und Hochschullehrer & 39 \\
\hline Lehrer des Sekundarbereiches mit akademischer Ausbildung & 4 \\
\hline Pädagogik- und Didaktikspezialisten & 1 \\
\hline Sonstige Lehrkräfte mit akademischer Ausbildung, anderweitig & 51 \\
\hline Unternehmensberatungs- und Organisationsfachkräfte, anderweitig & 11 \\
\hline Juristen, anderweitig nicht genannt & 1 \\
\hline Archiv- und Museumswissenschaftler & 2 \\
\hline Soziologen, Anthropologen und verwandte Wissenschaftler & 15 \\
\hline Philosophen, Historiker und Politologen & 12 \\
\hline Schriftsteller, bildende oder darstellende Künstler & 1 \\
\hline Autoren, Journalisten und andere Schriftsteller & 6 \\
\hline Bildhauer, Maler und verwandte Künstler & 1 \\
\hline Akademische Verwaltungsfachkräfte des öffentlichen Dienstes & 2 \\
\hline Techniker und gleichrangige nichttechnische Berufe & 3 \\
\hline Material- und ingenieurtechnische Fachkräfte & 2 \\
\hline Gesundheits-, Umweltschutzinspektoren und Qualitätskontrolle & 2 \\
\hline Land- und forstwirtschaftliche Berater & 1 \\
\hline Medizinische Assistenten & 1 \\
\hline Physiotherapeuten und verwandte Berufe & 1 \\
\hline Sonstige Lehrkräfte ohne akademische Ausbildung & 3 \\
\hline Technische und kaufmännische Handelsvertreter & 1 \\
\hline Finanz- und Verkaufsfachkräfte, anderweitig nicht genannt & 1 \\
\hline Verwaltungssekretäre und verwandte Fachkräfte & 5 \\
\hline Staatliche Steuer- und Abgabenbedienstete & 2 \\
\hline Sozialpflegerische Berufe & 1 \\
\hline Sonstige Büroangestellte & 7 \\
\hline Reiseführer & 1 \\
\hline Ladenverkäufer, Verkaufs-, Marktstandverkäufer und Vorführer & 1 \\
\hline Waldarbeiter und Holzfäller & 1 \\
\hline Total & 219 \\
\hline
\end{tabular}


Tabelle A6: Berufsbezeichnung der letzten Tätigkeit (ISCO-88-Codierung)

\begin{tabular}{|l|l|}
\hline & Abs. \\
\hline Produktions- und Operationsleiter, anderweitig nicht genannt & 1 \\
\hline Architekten, Raum- und Verkehrsplaner & 1 \\
\hline Pharmakologen, Pathologen und verwandte Wissenschaftler & 1 \\
\hline Universitäts- und Hochschullehrer & 3 \\
\hline Sonstige Lehrkräfte mit akademischer Ausbildung, anderweitig & 2 \\
\hline Unternehmensberatungs- und Organisationsfachkräfte, anderweitig & 2 \\
\hline Richter & 1 \\
\hline Juristen, anderweitig nicht genannt & 1 \\
\hline Soziologen, Anthropologen und verwandte Wissenschaftler & 2 \\
\hline Philologen, Übersetzer und Dolmetscher & 3 \\
\hline Autoren, Journalisten und andere Schriftsteller & 2 \\
\hline Film-, Bühnen- und sonstige Schauspieler, Regisseure & 1 \\
\hline Material- und ingenieurtechnische Fachkräfte & 1 \\
\hline Arbeits- und Personalvermittler & 1 \\
\hline Sekretariatskräfte & 1 \\
\hline Total & 23 \\
\hline
\end{tabular}

Tabelle A7: Paritätsverteilung nach Bildungsabschluss

\begin{tabular}{|l|l|l|l|}
\hline & $\begin{array}{l}\text { Nicht-Akademikerinnen } \\
(\mathrm{GGS})\end{array}$ & $\begin{array}{l}\text { Akademikerinnen } \\
(\mathrm{GGS})\end{array}$ & $\begin{array}{l}\text { Wissenschaftlerinnen } \\
\text { (Zusatzsample) }\end{array}$ \\
\hline 0 Kinder & $29 \%$ & $55 \%$ & $69 \%$ \\
\hline 1 Kind & $24 \%$ & $19 \%$ & $18 \%$ \\
\hline 2 Kinder & $32 \%$ & $21 \%$ & $11 \%$ \\
\hline $3+$ Kinder & $15 \%$ & $6 \%$ & $2 \%$ \\
\hline Total & $100 \%$ & $100 \%$ & $100 \%$ \\
\hline
\end{tabular}


Tabelle A8: Frauen nach Geburtsjahrgängen und Bildungsgruppen, absolut, ungewichtet

\begin{tabular}{|l|l|l|l|}
\hline & $\begin{array}{l}\text { Nicht-Akademikerinnen } \\
(\text { GGS })\end{array}$ & $\begin{array}{l}\text { Akademikerinnen } \\
\text { (GGS) }\end{array}$ & $\begin{array}{l}\text { Wissenschaftlerinnen } \\
\text { (Zusatzsample) }\end{array}$ \\
\hline 1963 & 65 & 5 & 0 \\
\hline 1964 & 113 & 16 & 3 \\
\hline 1965 & 136 & 23 & 2 \\
\hline 1966 & 132 & 25 & 6 \\
\hline 1967 & 118 & 16 & 7 \\
\hline 1968 & 109 & 22 & 9 \\
\hline 1969 & 113 & 14 & 9 \\
\hline 1970 & 118 & 8 & 8 \\
\hline 1971 & 101 & 15 & 10 \\
\hline 1972 & 120 & 12 & 11 \\
\hline 1973 & 78 & 10 & 7 \\
\hline 1974 & 97 & 26 & 14 \\
\hline 1975 & 82 & 28 & 16 \\
\hline 1976 & 92 & 21 & 10 \\
\hline 1977 & 74 & 20 & 20 \\
\hline 1978 & 81 & 16 & 21 \\
\hline 1979 & 76 & 16 & 21 \\
\hline 1980 & 95 & 12 & 17 \\
\hline 1981 & 89 & 12 & 29 \\
\hline 1982 & 105 & 14 & 12 \\
\hline 1983 & 81 & 16 & 2 \\
\hline Total & 2.075 & 347 & 234 \\
\hline & & & \\
\hline & & 21 & \\
\hline
\end{tabular}




\section{Codebuch für zusätzlich aufgenommene Fragen}

Die folgende Zusammenstellung beinhaltet jene Fragen, die zusätzlich zum Fragenblock des GGS aufgenommen wurden. Bei der Erstellung der zusätzlichen Fragen waren folgende Personen beteiligt: Bilal Barakat, Caroline Berghammer, Isabella Buber, Priska Flandorfer, Alexia Fürnkranz-Prskawetz, Paola di Giulio, Wolfgang Lutz, Tomáš Sobotka, Maria Rita Testa, Maria Winkler-Dworak, Kryštof Zeman.

\section{F828b01: früherer Tätigkeitsbereich}

„In welchem Bereich/welchen Bereichen waren Sie tätig?“

1 - Hochschulbereich, z.B. Universität oder Fachhochschule

2 - Außeruniversitäre Forschungseinrichtung

3 - Forschung und Entwicklung (F\&E) in einem privaten Institut/Unternehmen

4 - Privatwirtschaft (andere als F\&E Tätigkeit)

5 - Öffentliche Verwaltung

6 - Schule, Jugend- und Erwachsenenbildung

7 - Medien, Presse

8 - Non-Profit Organisationen (Hilfsorganisation, Gewerkschaft, politische Partei etc.)

9 - derzeit nicht berufstätig

10 - Sonstiges

\section{F828b02: früherer Tätigkeitsbereich}

„In welchem Bereich/welchen Bereichen waren Sie tätig?“6

1 - Hochschulbereich, z.B. Universität oder Fachhochschule

2 - Außeruniversitäre Forschungseinrichtung

3 - Forschung und Entwicklung (F\&E) in einem privaten Institut/Unternehmen

4 - Privatwirtschaft (andere als F\&E Tätigkeit)

5 - Öffentliche Verwaltung

6 - Schule, Jugend- und Erwachsenenbildung

7 - Medien, Presse

8 - Non-Profit Organisationen (Hilfsorganisation, Gewerkschaft, politische Partei etc.)

9 - derzeit nicht berufstätig

10 - Sonstiges

\section{F828b03: früherer Tätigkeitsbereich}

„In welchem Bereich/welchen Bereichen waren Sie tätig?“

1 - Hochschulbereich, z.B. Universität oder Fachhochschule

2 - Außeruniversitäre Forschungseinrichtung

3 - Forschung und Entwicklung (F\&E) in einem privaten Institut/Unternehmen

4 - Privatwirtschaft (andere als F\&E Tätigkeit)

5 - Öffentliche Verwaltung

6 - Schule, Jugend- und Erwachsenenbildung

7 - Medien, Presse

8 - Non-Profit Organisationen (Hilfsorganisation, Gewerkschaft, politische Partei etc.)

9 - derzeit nicht berufstätig

10 - Sonstiges

F828b04 bis F828b10: früherer Tätigkeitsbereich

Alles „Filter“"

F828b_tx: sonstiges

Bemerkung: Falls „Sonstiges“ in F828b01 
F828c - frühere berufliche Stellung

„Welche berufliche Stellung hatten Sie?“6

1 - Professorin

2 - a.o. Professorin

3 - Dozentin / Privatdozentin

4 - Assistentin

5 - Wissenschaftliche Mitarbeiterin

6 - Lektorin

7 - Sonstiges, bitte angeben:

F828c_tx: sonstiges

Bemerkung: Falls ,Sonstiges“ in F828c

F828d - frühere fachliche Ausrichtung

„Wie war die fachliche Ausrichtung Ihrer letzten Beschäftigung?“

1 - Naturwissenschaftlich

2 - Technisch

3 - Sozialwissenschaftlich / Pädagogisch

4 - Geisteswissenschaftlich

5 - Agrarwissenschaftlich

6 - Rechtswissenschaftlich

7 - Wirtschaftswissenschaftlich

8 - Medizinisch

9 - handwerklich / künstlerisch

F832b01: derzeitiger Tätigkeitsbereich

„In welchem Bereich/welchen Bereichen sind Sie tätig?

1 - Hochschulbereich, z.B. Universität oder Fachhochschule

2 - Außeruniversitäre Forschungseinrichtung

3 - Forschung und Entwicklung (F\&E) in einem privaten Institut/Unternehmen

4 - Privatwirtschaft (andere als F\&E Tätigkeit)

5 - Öffentliche Verwaltung

6 - Schule, Jugend- und Erwachsenenbildung

7 - Medien, Presse

8 - Non-Profit Organisationen (Hilfsorganisation, Gewerkschaft, politische Partei etc.)

9 - derzeit nicht berufstätig

10 - Sonstiges

F832b02: derzeitiger Tätigkeitsbereich

„In welchem Bereich/welchen Bereichen sind Sie tätig?

1 - Hochschulbereich, z.B. Universität oder Fachhochschule

2 - Außeruniversitäre Forschungseinrichtung

3 - Forschung und Entwicklung (F\&E) in einem privaten Institut/Unternehmen

4 - Privatwirtschaft (andere als F\&E Tätigkeit)

5 - Öffentliche Verwaltung

6 - Schule, Jugend- und Erwachsenenbildung

7 - Medien, Presse

8 - Non-Profit Organisationen (Hilfsorganisation, Gewerkschaft, politische Partei etc.)

9 - derzeit nicht berufstätig

10 - Sonstiges 
F832b03: derzeitiger Tätigkeitsbereich

„In welchem Bereich/welchen Bereichen sind Sie tätig?

1 - Hochschulbereich, z.B. Universität oder Fachhochschule

2 - Außeruniversitäre Forschungseinrichtung

3 - Forschung und Entwicklung (F\&E) in einem privaten Institut/Unternehmen

4 - Privatwirtschaft (andere als F\&E Tätigkeit)

5 - Öffentliche Verwaltung

6 - Schule, Jugend- und Erwachsenenbildung

7 - Medien, Presse

8 - Non-Profit Organisationen (Hilfsorganisation, Gewerkschaft, politische Partei etc.)

9 - derzeit nicht berufstätig

10 - Sonstiges

F832b04: derzeitiger Tätigkeitsbereich

„In welchem Bereich/welchen Bereichen sind Sie tätig?

1 - Hochschulbereich, z.B. Universität oder Fachhochschule

2 - Außeruniversitäre Forschungseinrichtung

3 - Forschung und Entwicklung (F\&E) in einem privaten Institut/Unternehmen

4 - Privatwirtschaft (andere als F\&E Tätigkeit)

5 - Öffentliche Verwaltung

6 - Schule, Jugend- und Erwachsenenbildung

7 - Medien, Presse

8 - Non-Profit Organisationen (Hilfsorganisation, Gewerkschaft, politische Partei etc.)

9 - derzeit nicht berufstätig

10 - Sonstiges

\section{F832b05: derzeitiger Tätigkeitsbereich}

„In welchem Bereich/welchen Bereichen sind Sie tätig?

1 - Hochschulbereich, z.B. Universität oder Fachhochschule

2 - Außeruniversitäre Forschungseinrichtung

3 - Forschung und Entwicklung (F\&E) in einem privaten Institut/Unternehmen

4 - Privatwirtschaft (andere als F\&E Tätigkeit)

5 - Öffentliche Verwaltung

6 - Schule, Jugend- und Erwachsenenbildung

7 - Medien, Presse

8 - Non-Profit Organisationen (Hilfsorganisation, Gewerkschaft, politische Partei etc.)

9 - derzeit nicht berufstätig

10 - Sonstiges

\section{F832b06: derzeitiger Tätigkeitsbereich}

„In welchem Bereich/welchen Bereichen sind Sie tätig?

1 - Hochschulbereich, z.B. Universität oder Fachhochschule

2 - Außeruniversitäre Forschungseinrichtung

3 - Forschung und Entwicklung (F\&E) in einem privaten Institut/Unternehmen

4 - Privatwirtschaft (andere als F\&E Tätigkeit)

5 - Öffentliche Verwaltung

6 - Schule, Jugend- und Erwachsenenbildung

7 - Medien, Presse

8 - Non-Profit Organisationen (Hilfsorganisation, Gewerkschaft, politische Partei etc.)

9 - derzeit nicht berufstätig

10 - Sonstiges 
F832b07 bis F832b10: derzeitiger Tätigkeitsbereich

Alles ,Filter“

F832b_tx: sonstiges

Bemerkung: Falls „Sonstiges“ in F832b01

F832c - derzeitige berufliche Stellung

„Welche berufliche Stellung haben Sie?“

1 - Professorin

2 - a.o. Professorin

3 - Dozentin / Privatdozentin

4 - Assistentin

5 - Wissenschaftliche Mitarbeiterin

6 - Lektorin

7 - Sonstiges, bitte angeben:

F832c tx: sonstiges

Bemerkung: Falls „Sonstiges“ in F832b01

F832d: derzeitige fachliche Ausrichtung

„Wie ist die fachliche Ausrichtung Ihrer derzeitigen Beschäftigung?“

1 - Naturwissenschaftlich

2 - Technisch

3 - Sozialwissenschaftlich / Pädagogisch

4 - Geisteswissenschaftlich

5 - Agrarwissenschaftlich

6 - Rechtswissenschaftlich

7 - Wirtschaftswissenschaftlich

8 - Medizinisch

9 - handwerklich / künstlerisch

F845c1: Befristung

„Auf insgesamt wie viele Jahre und Monate ist Ihr derzeitiger Vertrag befristet?“

1 - Monat(e)

$2-\operatorname{Jahr}(\mathrm{e})$

3 - Weiß nicht

F845c2: Befristung

F845c3: Befristung

Beide Variablen nicht belegt, alles „Filter“

F845_M: Befristung Monate

Monate

F845_J: Befristung Jahre

Jahre 
F845d: Aufsicht auf unbefristete Stelle

„Haben Sie Aussicht auf eine unbefristete Stelle in den nächsten drei Jahren?“

1 - ganz sicher ja

2 - wahrscheinlich ja

3 - wahrscheinlich nicht

4 - ganz sicher nicht

5 - weiß nicht

F856a: Probleme Arbeitsplatz zu finden

„Hatten Sie jemals über einen Zeitraum von 6 Monaten oder länger Probleme, einen Arbeitsplatz zu finden?“

$$
\begin{aligned}
& 1-\mathrm{Ja} \\
& 2-\text { Nein }
\end{aligned}
$$

F856b: Arbeitsplatzsicherheit

„Wie sicher oder unsicher war Ihr Arbeitsplatz in den letzten 3 Jahren?“

1 - Sehr sicher

2 - Sicher

3 - Weder noch

4 - Unsicher

5 - Sehr unsicher

F856c: Auslandsaufenthalt

„Waren Sie bereits mindestens ein Mal für drei Monate oder länger aus (eigenen) beruflichen Gründen oder im Rahmen einer postsekundären Ausbildung im Ausland?“

$1-\mathrm{Ja}$

$2-$ Nein

F856d: Zahl Auslandsaufenthalte

„Wieviele Aufenthalte hatten Sie?"

Aufenthalte

F856eJ: Jahre Auslandsaufenthalte

„Was ist die Gesamtdauer dieser Aufenthalte?“ Jahre

F856eM: Monate Auslandsaufenthalte

„Was ist die Gesamtdauer dieser Aufenthalte?“

und Monate

F856f: Ausland: Auswirkung auf Partnerschaft „Wie waren die Auswirkungen auf Ihre (damalige) Partnerschaft im Zusammenhang mit diesem/n Aufenthalt/en?"6

1 - Sehr negative Auswirkungen

2 - Geringe negative Auswirkungen

3 - Keine Auswirkungen

4 - Geringe positive Auswirkungen

5 - Sehr positive Auswirkungen

6 - hatte keinen Partner 
F856g: Ausland: Auswirkung auf Familienplanung

„Wie waren die Auswirkungen auf Ihre Familienplanung im Zusammenhang mit diesem/n Aufenthalt/en Auswirkungen?“6

1 - Sehr negative Auswirkungen

2 - Geringe negative Auswirkungen

3 - Keine Auswirkungen

4 - Geringe positive Auswirkungen

5 - Sehr positive Auswirkungen

F856h: Auslandsaufenthalt geplant?

„Haben Sie vor, innerhalb der nächsten 3 Jahre einen mindestens 3-monatigen Auslandsaufenthalt aus (eigenen) beruflichen Gründen zu absolvieren?“‘

1 - Ganz sicher ja

2 - wahrscheinlich ja

3 - wahrscheinlich nicht

4 - ganz sicher nicht

F856i: Häufigkeit mehrtägiger Tagungen etc.

„Wie oft waren Sie in den letzten drei Jahren aufgrund von Konferenzen, Tagungen und ähnlichem mehrere Tage außer Haus?““

pro Jahr

$-2-$ weiß nicht

F856j01: Kinderbetreuung durch..

„Wer übernimmt in dieser Zeit die Kinderbetreuung? oder: Wer übernimmt überwiegend die Kinderbetreuung?“ (Wenn Kinder unter 12 Jahren)

$$
\begin{aligned}
& 1 \text { - (Ehe-)Partner } \\
& 2 \text { - Ex-Partner } \\
& 3 \text { - Mutter } \\
& 4 \text { - Vater } \\
& 5 \text { - Stiefmutter } \\
& 7 \text { - Mutter des (Ehe-)Partners } \\
& 8 \text { - Vater des (Ehe-)Partners } \\
& 22 \text { - Freunde, Bekannte, Nachbarn, Kollegen } \\
& 20 \text { - Bruder von Ihnen bzw. des (Ehe-)Partners } \\
& 24 \text { - andere Person }
\end{aligned}
$$

F856j02: Kinderbetreuung durch..

„Wer übernimmt in dieser Zeit die Kinderbetreuung? oder: Wer übernimmt überwiegend die Kinderbetreuung?" (Wenn Kinder unter 12 Jahren)

$$
\begin{aligned}
& 1 \text { - (Ehe-)Partner } \\
& 2 \text { - Ex-Partner } \\
& 3 \text { - Mutter } \\
& 4 \text { - Vater } \\
& 5 \text { - Stiefmutter } \\
& 7 \text { - Mutter des (Ehe-)Partners } \\
& 8 \text { - Vater des (Ehe-)Partners } \\
& 22 \text { - Freunde, Bekannte, Nachbarn, Kollegen } \\
& 20 \text { - Bruder von Ihnen bzw. des (Ehe-)Partners } \\
& 24 \text { - andere Person }
\end{aligned}
$$


F856j03: Kinderbetreuung durch..

„Wer übernimmt in dieser Zeit die Kinderbetreuung? oder: Wer übernimmt überwiegend die Kinderbetreuung?" (Wenn Kinder unter 12 Jahren)

$$
\begin{aligned}
& 1 \text { - (Ehe-)Partner } \\
& 2 \text { - Ex-Partner } \\
& 3 \text { - Mutter } \\
& 4 \text { - Vater } \\
& 5 \text { - Stiefmutter } \\
& 7 \text { - Mutter des (Ehe-)Partners } \\
& 8 \text { - Vater des (Ehe-)Partners } \\
& 22 \text { - Freunde, Bekannte, Nachbarn, Kollegen } \\
& 20 \text { - Bruder von Ihnen bzw. des (Ehe-)Partners } \\
& 24 \text { - andere Person }
\end{aligned}
$$

F856j04: Kinderbetreuung durch..

„Wer übernimmt in dieser Zeit die Kinderbetreuung? oder: Wer übernimmt überwiegend die Kinderbetreuung?“ (Wenn Kinder unter 12 Jahren)

$$
\begin{aligned}
& 1 \text { - (Ehe-)Partner } \\
& 2 \text { - Ex-Partner } \\
& 3 \text { - Mutter } \\
& 4 \text { - Vater } \\
& 5 \text { - Stiefmutter } \\
& 7 \text { - Mutter des (Ehe-)Partners } \\
& 8 \text { - Vater des (Ehe-)Partners } \\
& 22 \text { - Freunde, Bekannte, Nachbarn, Kollegen } \\
& 20 \text { - Bruder von Ihnen bzw. des (Ehe-)Partners } \\
& 24 \text { - andere Person }
\end{aligned}
$$

F856j05: Kinderbetreuung durch..

„Wer übernimmt in dieser Zeit die Kinderbetreuung? oder: Wer übernimmt überwiegend die Kinderbetreuung?" (Wenn Kinder unter 12 Jahren)

$$
\begin{aligned}
& 1 \text { - (Ehe-)Partner } \\
& 2 \text { - Ex-Partner } \\
& 3 \text { - Mutter } \\
& 4 \text { - Vater } \\
& 5 \text { - Stiefmutter } \\
& 7 \text { - Mutter des (Ehe-)Partners } \\
& 8 \text { - Vater des (Ehe-)Partners } \\
& 22 \text { - Freunde, Bekannte, Nachbarn, Kollegen } \\
& 20 \text { - Bruder von Ihnen bzw. des (Ehe-)Partners } \\
& 24 \text { - andere Person }
\end{aligned}
$$


F856j06: Kinderbetreuung durch..

„Wer übernimmt in dieser Zeit die Kinderbetreuung? oder: Wer übernimmt überwiegend die Kinderbetreuung?" (Wenn Kinder unter 12 Jahren)

$$
\begin{aligned}
& 1 \text { - (Ehe-)Partner } \\
& 2 \text { - Ex-Partner } \\
& 3 \text { - Mutter } \\
& 4 \text { - Vater } \\
& 5 \text { - Stiefmutter } \\
& 7 \text { - Mutter des (Ehe-)Partners } \\
& 8 \text { - Vater des (Ehe-)Partners } \\
& 22 \text { - Freunde, Bekannte, Nachbarn, Kollegen } \\
& 20 \text { - Bruder von Ihnen bzw. des (Ehe-)Partners } \\
& 24 \text { - andere Person }
\end{aligned}
$$

F856j07 bis F856j24: Kinderbetreuung durch..

Alles „Filter“

F856k: Problem mit Organisieren der Kinderbetreuung

„Wie oft waren diese Aufenthalte ein Problem für das Organisieren der Kinderbetreuung?“"

Bemerkung: Wenn Kinder unter 12 Jahren.

$$
\begin{aligned}
& \text { 1- Immer } \\
& 2 \text { - Oft } \\
& 3 \text { - Manchmal } \\
& 4 \text { - Selten } \\
& 5 \text { - Nie }
\end{aligned}
$$

F917b01: P: früherer Tätigkeitsbereich „In welchem Bereich/welchen Bereichen war Ihr Partner tätig?““



F917b02 bis F917b10: P: früherer Tätigkeitsbereich

Analog angelegt, nur „Filter“ codiert

F917b_tx: sonstiges

Bemerkung: Falls „Sonstiges“ in F917b01

F917c: P: frühere berufliche Stellung

Bemerkung: nur „Filter“

F917c tx: sonstiges

nur „Filter“ 
F921b01: P: derzeitiger Tätigkeitsbereich

„In welchem Bereich/welchen Bereichen ist Ihr Partner tätig?““

1 - Hochschulbereich, z.B. Universität oder Fachhochschule

2 - Außeruniversitäre Forschungseinrichtung

3 - Forschung und Entwicklung (F\&E) in einem privaten Institut/Unternehmen

4 - Privatwirtschaft (Andere als F\&E )

5 - Öffentliche Verwaltung

6 - Schule, Jugend- und Erwachsenenbildung

7 - Medien, Presse

8 - Non-Profit Organisationen (Hilfsorganisation, Gewerkschaft, politische Partei etc.)

9 - derzeit nicht berufstätig

10 - Sonstiges

F921b02: P: derzeitiger Tätigkeitsbereich

„In welchem Bereich/welchen Bereichen ist Ihr Partner tätig?““

1 - Hochschulbereich, z.B. Universität oder Fachhochschule

2 - Außeruniversitäre Forschungseinrichtung

3 - Forschung und Entwicklung (F\&E) in einem privaten Institut/Unternehmen

4 - Privatwirtschaft (Andere als F\&E )

5 - Öffentliche Verwaltung

6 - Schule, Jugend- und Erwachsenenbildung

7 - Medien, Presse

8 - Non-Profit Organisationen (Hilfsorganisation, Gewerkschaft, politische Partei etc.)

9 - derzeit nicht berufstätig

10 - Sonstiges

F921b03: P: derzeitiger Tätigkeitsbereich

„In welchem Bereich/welchen Bereichen ist Ihr Partner tätig?““

1 - Hochschulbereich, z.B. Universität oder Fachhochschule

2 - Außeruniversitäre Forschungseinrichtung

3 - Forschung und Entwicklung (F\&E) in einem privaten Institut/Unternehmen

4 - Privatwirtschaft (Andere als F\&E )

5 - Öffentliche Verwaltung

6 - Schule, Jugend- und Erwachsenenbildung

7 - Medien, Presse

8 - Non-Profit Organisationen (Hilfsorganisation, Gewerkschaft, politische Partei etc.)

9 - derzeit nicht berufstätig

10 - Sonstiges

F921b04: P: derzeitiger Tätigkeitsbereich

„In welchem Bereich/welchen Bereichen ist Ihr Partner tätig?““

1 - Hochschulbereich, z.B. Universität oder Fachhochschule

2 - Außeruniversitäre Forschungseinrichtung

3 - Forschung und Entwicklung (F\&E) in einem privaten Institut/Unternehmen

4 - Privatwirtschaft (Andere als F\&E )

5 - Öffentliche Verwaltung

6 - Schule, Jugend- und Erwachsenenbildung

7 - Medien, Presse

8 - Non-Profit Organisationen (Hilfsorganisation, Gewerkschaft, politische Partei etc.)

9 - derzeit nicht berufstätig

10 - Sonstiges 
F921b05 bis F921b10: P: derzeitiger Tätigkeitsbereich

Analog angelegt, nur „Filter“ codiert

F921_tx: sonstiges

Bemerkung: Falls „Sonstiges“ in F921b01

F921c: P: derzeitige berufliche Stellung

„Welche berufliche Stellung hat er?““

$$
\begin{aligned}
& 1 \text { - Professor } \\
& 2 \text { - a.o. Professor } \\
& 3 \text { - Dozent / Privatdozent } \\
& 4 \text { - Assistent } \\
& 5 \text { - Wissenschaftliche Mitarbeiter } \\
& 6 \text { - Lektor } \\
& 7 \text { - Sonstiges }
\end{aligned}
$$

F921c_tx: sonstiges

Bemerkung: Falls ,Sonstiges“ in F921c

$$
\begin{gathered}
\text { F1115_tx: Einleitungstext } \\
1-\text { weiter } \\
-3-\text { Filter }
\end{gathered}
$$

F1115_1: Auswirkungen: Auslandsaufenthalt

„Die Vereinbarkeit von Familie und Beruf kann auch von äußeren Umständen beeinflusst werden. Ich nenne Ihnen nun einige Einflussfaktoren. Wie sind für Sie persönlich die Auswirkungen auf die Vereinbarkeit von Familie und Beruf?“‘

Auslandsaufenthalt

$$
\begin{aligned}
& 1 \text { - Sehr negative Auswirkungen } \\
& 2 \text { - Geringe negative Auswirkungen } \\
& 3 \text { - Keine Auswirkungen } \\
& 4 \text { - Geringe positive Auswirkungen } \\
& 5 \text { - Sehr positive Auswirkungen } \\
& 6 \text { - Trifft nicht zu }
\end{aligned}
$$

F1115_2: Auswirkungen: lange Arbeitszeiten „Die Vereinbarkeit von Familie und Beruf kann auch von äußeren Umständen beeinflusst werden. Ich nenne Ihnen nun einige Einflussfaktoren. Wie sind für Sie persönlich die Auswirkungen auf die Vereinbarkeit von Familie und Beruf?“‘

Lange Arbeitszeiten

1 - Sehr negative Auswirkungen

2 - Geringe negative Auswirkungen

3 - Keine Auswirkungen

4 - Geringe positive Auswirkungen

5 - Sehr positive Auswirkungen

6 - Trifft nicht $\mathrm{zu}$ 
F1115_3: Auswirkungen: unregelmäßige Arbeitszeiten

„Die Vereinbarkeit von Familie und Beruf kann auch von äußeren Umständen beeinflusst werden. Ich nenne Ihnen nun einige Einflussfaktoren. Wie sind für Sie persönlich die Auswirkungen auf die Vereinbarkeit von Familie und Beruf?“"

Unregelmäßige Arbeitszeiten

$$
\begin{aligned}
& 1 \text { - Sehr negative Auswirkungen } \\
& 2 \text { - Geringe negative Auswirkungen } \\
& 3 \text { - Keine Auswirkungen } \\
& 4 \text { - Geringe positive Auswirkungen } \\
& 5 \text { - Sehr positive Auswirkungen } \\
& 6 \text { - Trifft nicht zu }
\end{aligned}
$$

F1115_4: Auswirkungen: flexible Arbeitszeiten „Die Vereinbarkeit von Familie und Beruf kann auch von äußeren Umständen beeinflusst werden. Ich nenne Ihnen nun einige Einflussfaktoren. Wie sind für Sie persönlich die Auswirkungen auf die Vereinbarkeit von Familie und Beruf?“"

Flexible Arbeitszeiten

$$
\begin{aligned}
& 1 \text { - Sehr negative Auswirkungen } \\
& 2 \text { - Geringe negative Auswirkungen } \\
& 3 \text { - Keine Auswirkungen } \\
& 4 \text { - Geringe positive Auswirkungen } \\
& 5 \text { - Sehr positive Auswirkungen } \\
& 6 \text { - Trifft nicht zu }
\end{aligned}
$$

F1115_5: Auswirkungen: Verbleiben am aktuellen Wissensstand „Die Vereinbarkeit von Familie und Beruf kann auch von äußeren Umständen beeinflusst werden. Ich nenne Ihnen nun einige Einflussfaktoren. Wie sind für Sie persönlich die Auswirkungen auf die Vereinbarkeit von Familie und Beruf?"“

Verbleiben am aktuellen Wissensstand

$$
\begin{aligned}
& 1 \text { - Sehr negative Auswirkungen } \\
& 2 \text { - Geringe negative Auswirkungen } \\
& 3 \text { - Keine Auswirkungen } \\
& 4 \text { - Geringe positive Auswirkungen } \\
& 5 \text { - Sehr positive Auswirkungen } \\
& 6 \text { - Trifft nicht zu }
\end{aligned}
$$

F1115_6: Auswirkungen: Unterstützung durch Partner „Die Vereinbarkeit von Familie und Beruf kann auch von äußeren Umständen beeinflusst werden. Ich nenne Ihnen nun einige Einflussfaktoren. Wie sind für Sie persönlich die Auswirkungen auf die Vereinbarkeit von Familie und Beruf?“"

Unterstützung durch Partner

1 - Sehr negative Auswirkungen

2 - Geringe negative Auswirkungen

3 - Keine Auswirkungen

4 - Geringe positive Auswirkungen

5 - Sehr positive Auswirkungen

6 - Trifft nicht zu 
F1115_7: Auswirkungen: Angebot von Kinderbetreuung durch Arbeitgeber „Die Vereinbarkeit von Familie und Beruf kann auch von äußeren Umständen beeinflusst werden. Ich nenne Ihnen nun einige Einflussfaktoren. Wie sind für Sie persönlich die Auswirkungen auf die Vereinbarkeit von Familie und Beruf?"“

Angebot von Kinderbetreuung von Seiten des Arbeitgebers

$$
\begin{aligned}
& 1 \text { - Sehr negative Auswirkungen } \\
& 2 \text { - Geringe negative Auswirkungen } \\
& 3 \text { - Keine Auswirkungen } \\
& 4 \text { - Geringe positive Auswirkungen } \\
& 5 \text { - Sehr positive Auswirkungen } \\
& 6 \text { - Trifft nicht zu }
\end{aligned}
$$

F1116: weitere Faktoren?

„Gibt es diesbezüglich noch andere Faktoren?“

$$
\begin{aligned}
& 1 \text { - ja } \\
& 2 \text { - nein }
\end{aligned}
$$

F1116_tx: weitere Faktoren

Bemerkung: Texteintrag, falls F1116=ja

F1117: Mutter und Karriere „Als wie leicht oder schwer schätzen Sie es ein, in dem Bereich, in dem Sie arbeiten, als Mutter Karriere zu machen?“6

$$
\begin{aligned}
& 1 \text { - Sehr schwer } \\
& 2 \text { - Schwer } \\
& 3 \text { - Weder/noch } \\
& 4 \text { - Leicht } \\
& 5 \text { - Sehr leicht }
\end{aligned}
$$

F1118: berufliche Ziele und Familie

„Für Sie persönlich, als wie leicht oder schwer schätzen Sie es ein, die Ziele, die Sie beruflich anstreben, mit Familie zu vereinbaren?“"

$$
\begin{aligned}
& 1 \text { - Sehr schwer } \\
& 2 \text { - Schwer } \\
& 3 \text { - Weder/noch } \\
& 4 \text { - Leicht } \\
& 5 \text { - Sehr leicht }
\end{aligned}
$$

F1119: Familienplanung geändert?

„Haben Sie jemals aus beruflichen Gründen Ihre Familienplanung geändert?“

$$
\begin{aligned}
& 1 \text { - Ja } \\
& 2 \text { - Nein } \\
& 3 \text { - Trifft nicht zu }
\end{aligned}
$$

F1120: Familienplanung verschoben?

„Haben Sie Ihre Familienplanung verschoben?“

$$
\begin{aligned}
& 1 \text { - Ja } \\
& 2 \text { - Nein } \\
& 3 \text { - Trifft nicht zu }
\end{aligned}
$$


F1121: gewünschte Kinderzahl reduziert?

„Haben Sie Ihre gewünschte Kinderzahl reduziert?“

$1-\mathrm{Ja}$

$2-$ Nein

3 - Trifft nicht zu

„Die folgenden Fragen beziehen sich auf Familie und Beruf:“6

F1122: Wünsche Politik

Welche Wünsche haben Sie diesbezüglich an die Politik?‘،

F1123: Wünsche Wissenschaftsmanagement

„Welche Wünsche haben Sie diesbezüglich an das Wissenschaftsmanagement (Universitäten, Österreichische Akademie der Wissenschaften)?“‘

F1124: Wünsche Firmen

„Welche Wünsche haben Sie diesbezüglich an Firmen, die Wissenschaftlerinnen beschäftigen?““

F1125: Wünsche Wissenschaftsministerium

„Welche Wünsche haben Sie diesbezüglich an das Wissenschaftsministerium?““

Informationen der Stipendienstelle der ÖAW, die inkludiert wurden

Antragsjahr1: Jahr der Antragstellung für 1. Stipendium

1993-2007

Stip1: Art der beantragten Förderung

1 - Apart

$2-\mathrm{DOC}$

3 - DOC-TEAM

4 - DOC-fForte

5 - Rom

Status1: Status der 1. Antragstellung

1 - Altstipendiatin

2 - Antragstellerin

3 - Stipendiatin

Phase1: Phase der 1. Antragstellung

1 - Abbruch

2 - Ablehnung formal

3 - Stipendium zurückgelegt

4 - Stipendium beendet

5 - Warteliste

6 - negativ beurteilt

7 - nicht begutachtet

8 - positiv beurteilt

9 - zurückgezogen 
Beginn_1: Beginn des 1. Stipendiums (Jahr)

1993-2008

Ende_1: Ende des 1. Stipendiums (Jahr)

1996-2011

Antragsjahr2: Jahr der Antragstellung für 2. Stipendium

1995-2007

Stip2: Art der beantragten Förderung

1 - Apart

$2-\mathrm{DOC}$

3 - DOC-TEAM

4 - DOC-fForte

5 - Rom

Status2: Status der 2. Antragstellung

1 - Altstipendiatin

2 - Antragstellerin

3 - Stipendiatin

Phase2: Phase der 2. Antragstellung

1 - Abbruch

2 - Ablehnung formal

3 - Stipendium zurückgelegt

4 - Stipendium beendet

5 - Warteliste

6 - negativ beurteilt

7 - nicht begutachtet

8 - positiv beurteilt

9 - zurückgezogen

Beginn_2: Beginn des 2. Stipendiums (Jahr)

1996-2008

Ende_2: Ende des 2. Stipendiums (Jahr)

1998-2010

Antragsjahr3: Jahr der Antragstellung für 3. Stipendium 2001-2007

Stip3: Art der beantragten Förderung

1 - Apart

$2-\mathrm{DOC}$

3 - DOC-TEAM

4 - DOC-fForte

5 - Rom 
Status3: Status der 3. Antragstellung

1 - Altstipendiatin

2 - Antragstellerin

3 - Stipendiatin

Phase3: Phase der 3. Antragstellung

1 - Abbruch

2 - Ablehnung formal

3 - Stipendium zurückgelegt

4 - Stipendium beendet

5 - Warteliste

6 - negativ beurteilt

7 - nicht begutachtet

8 - positiv beurteilt

9 - zurückgezogen

Beginn 3: Beginn des 3. Stipendiums (Jahr)

2001-2002

Ende_3: Ende des 3. Stipendiums (Jahr)

2003-2004

Antragsjahr4: Jahr der Antragstellung für 4. Stipendium 2005

Stip4: Art der beantragten Förderung

1 - Apart

$2-\mathrm{DOC}$

3 - DOC-TEAM

4 - DOC-fForte

5 - Rom

Status4: Status der 4. Antragstellung

1 - Altstipendiatin

2 - Antragstellerin

3 - Stipendiatin

Phase4: Phase der 4. Antragstellung

1 - Abbruch

2 - Ablehnung formal

3 - Stipendium zurückgelegt

4 - Stipendium beendet

5 - Warteliste

6 - negativ beurteilt

7 - nicht begutachtet

8 - positiv beurteilt

9 - zurückgezogen 


\section{VIENNA INSTITUTE OF DEMOGRAPHY}

\section{Working Papers}

Kuhn, Michael, Stefan Wrzaczek, Alexia Prskawetz, and Gustav Feichtinger, Externalities in a Life-Cycle Model with Endogenous Survival, VID Working Paper $01 / 2010$

Prettner, Klaus, Population Ageing and Endogenous Economic Growth, VID Working Paper 08/2009.

Št’astná, Anna and Tomáš Sobotka, Changing Parental Leave and Shifts in Second and Third-Birth Rates in Austria, VID Working Paper 07/2009.

Prettner, Klaus and Alexia Prskawetz, Decreasing Fertility, Economic Growth and the Intergenerational Wage Gap, VID Working Paper 06/2009.

Wegner, Christian and Marc Luy, Determinants of General Health and Specific Diseases of Elderly Women and Men: A Longitudinal Analysis for Western and Eastern Germany, VID Working Paper 05/2009.

Wrzaczek, Stefan, Michael Kuhn, Alexia Prskawetz, and Gustav Feichtinger, The Reproductive Value in Distributed Optimal Control Models, VID Working Paper $04 / 2009$.

Wendland, Maike und Isabella Buber, Ein Vergleich der Ergebnisse der ersten Welle des Österreichischen,, Survey of Health, Ageing and Retirement in Europe“ (SHARE) und der „Österreichischen Gesundheitsbefragung“ (ATHIS), VID Working Paper $03 / 2009$.

Thomson, Elizabeth, Maria Winkler-Dworak, Martin Spielauer, and Alexia Prskawetz, Union Instability as an Engine of Fertility, VID Working Paper 02/2009.

Grafeneder-Weissteiner, Theresa and Klaus Prettner, Agglomeration and population ageing in a two region model of exogenous growth, VID Working Paper 01/2009.

Skirbekk, Vegard, Anne Goujon, and Eric Kaufmann, Secularism or Catholicism? The Religious Composition of the United States to 2043, VID Working Paper $04 / 2008$.

Ediev, Dalkhat M., Extrapolative Projections of Mortality: Towards a More Consistent Method, VID Working Paper 03/2008.

The Vienna Institute of Demography Working Paper Series receives only limited review. Views or opinions expressed herein are entirely those of the authors. 